\begin{tabular}{|r|l|}
\hline \multicolumn{2}{|c|}{ Statistica Sinica Preprint No: SS-2021-0183 } \\
\hline Title & Functional Finite Mixture Regression Models \\
\hline Uanuscript ID & SS-2021-0183 \\
\hline DRL & http://www.stat.sinica.edu.tw/statistica/ \\
\hline DOmplete List of Authors & $\begin{array}{l}\text { Xiao Wang, } \\
\text { Yufeng Liu and } \\
\text { Hongtu Zhu }\end{array}$ \\
\hline Corresponding Author & Hongtu Zhu \\
\hline E-mail & htzhu@email.unc.edu \\
\hline Notice: Accepted version subject to English editing. \\
\hline
\end{tabular}


Statistica Sinica

\title{
FUNCTIONAL FINITE MIXTURE REGRESSION MODELS
}

\author{
Xiao Wang, Leo Yu-Feng Liu, and Hongtu Zhu \\ Department of Statistics \\ Purdue University, Indiana, U.S.A. \\ Department of Statistics and Operations Research \\ University of North Carolina at Chapel Hill, North Carolina, U.S.A. \\ Department of Biostatistics \\ University of North Carolina at Chapel Hill, North Carolina, U.S.A.
}

Abstract:

The aim of this paper is to develop a set of functional finite mixture regression models with functional predictors in the framework of the reproducing kernel Hilbert space. First, we show the consistency of a penalized likelihood model order estimator for the true model order, denoted as $q^{*}$. We further show that the penalty of order $q^{\frac{2 r}{2 r+1}} n^{\frac{1}{2 r+1}} \log (n)$ yields the strong consistent estimator of $q^{*}$, where $n$ and $q$ are the sample size and the model order, respectively, and $r$ is the eigenvalue decay rate of an operator determined jointly by the reproducing and covariance kernels. Second, we establish the minimax rate of convergence for the estimation risk. It is shown that the optimal rate is determined by the alignment of the reproducing kernel and the covariance kernel and the true model order $q^{*}$. 


\begin{abstract}
An efficient algorithm is also developed to estimate all unknown components of the functional finite mixture model. Simulation studies and a real data analysis are carried out to illustrate the merits of our method.
\end{abstract}

\title{
1. Introduction
}

With the rapid growth of modern technology, many large-scale biomedical studies (e.g., UK Biobank) have been/are being conducted to collect massive datasets with large volumes of multi-modality imaging, genetic, neurocognitive, and clinical information from increasingly large cohorts. Simultaneously extracting and integrating rich and diverse heterogeneous information in neuroimaging and/or other variables from these big data sets could transform our understanding of how diseases impact the structure and function of the brain and cognitive functions across the lifespan. Therefore, it is imperative to develop new learning methods (Liu and Zhu, 2021; Feng et al., 2020; Wang et al., 2021; Ombao et al., 2016), which are applicable to many neuroimaging studies for neuropsychiatric disorders, major neurodegenerative diseases, and normal brain development.

Mixture models is a powerful probabilistic model for using mixture distributions to represent the presence of subpopulations within an overall population. See a comprehensive review of the theory and applications of mix- 
ture models in McLachlan and Peel (2000). The most well-known mixture model is the Gaussian mixture model (Richard and Green, 1997), in which the density of a random vector $Y$ is represented by $p(y)=\sum_{i=1}^{q} \phi_{i} N\left(\mu_{i}, \Sigma_{i}\right)$, where the $i$-th vector component is characterized by a normal distribution with a weight $\phi_{i}$, a mean $\mu_{i}$, and a covariance $\Sigma_{i}$. This framework can be extended naturally to the regression setting. The Gaussian mixture regression model (Ghahramani and Jordan, 1994; Calinon et al., 2007; Stulp and Sigaud, 2015) assumes that the conditional distribution of multiple response responses given one or more predictors follows a Gaussian mixture distribution and the conditional mean is a function of the predictors. In this paper, we investigate major mathematical challenges in the conditional analysis of clinical response variables given ultra high-dimensional imaging predictors under the framework of functional finite mixture regression models.

Functional data analysis has been an active area of research, and wellknown monographs in this area include Ramsay and Sloverman (2005), Bowman (2010), and Ferraty and Vieu (2006). Functional regression, particularly the functional linear regression model, has been extensively studied. The literature on functional linear regression is vast. For example, scalar-on-function regression (a continuous response variable regressed on functional covariates), which corresponds to our proposed mixture model 
(1.1) with a single component, has been studied by (Cai and Hall, 2006, Crambes et al., 2009; Wang et al., 2016; Yuan and Cai, 2010; Hall and Horowitz, 2007; Du and Wang, 2014; Wang et al., 2017). When the true model is the finite mixture model with at least two different components, the standard functional linear regression will provide a poor result. There are few papers on the development of numerical algorithm for functional finite mixture regression models. Yao et al. (2011) proposed a class of functional regression models that allow the regression structure to vary for different subpopulations. By projecting the functional predictor process onto its eigenspace, the new functional finite mixture regression model is simplified to a framework that is similar to classical mixture regression models. However, none of them considered the reproducing kernel Hilbert space (RKHS) and investigated the theoretical properties of the RKHS estimates, particularly the minimax rate.

Consider a functional finite mixture regression model such that the conditional distribution of a scalar response $Y$ given a functional predictor $\{X(t): t \in \mathcal{I}\}$ belongs to the class of convex combination of $q^{*}$ densities 
given by

$$
\begin{gathered}
\mathcal{M}_{q^{*}}=\left\{f^{*}(y \mid x)=\sum_{k=1}^{q^{*}} \pi_{k}^{*} f_{0}\left(y-\left\langle x, \beta_{k}^{*}\right\rangle\right):\right. \\
\left.\pi_{k}^{*} \geq 0, \sum_{k=1}^{q^{*}} \pi_{k}^{*}=1, \beta_{k}^{*} \in \mathcal{F}\right\},
\end{gathered}
$$

where $f_{0}$ is fixed probability density (e.g., Gaussian) on $\mathbb{R}$ and $\langle x, \beta\rangle=$ $\int_{\mathcal{I}} x(t) \beta(t) d t$. The intercept terms can be incorporated into the model by writing each component as $f_{0}\left(y-\beta_{0 k}^{*}-\left\langle x, \beta_{k}^{*}\right\rangle\right)$, where the $\beta_{0 k}^{*}$ are intercepts. We ignore them in the following analysis only for ease of presentation. Here $\mathcal{F}$ is assumed to be a reproducing kernel Hilbert space (RKHS) with the reproducing kernel $K$. The RKHS $\mathcal{F}$ is a linear functional space endowed with an inner product $\langle\cdot, \cdot\rangle_{K}$ such that for any $t \in \mathcal{I}$, $K(t, \cdot) \in \mathcal{F}$, and $f(t)=\langle K(t, \cdot), f\rangle_{K}$ holds for any $f \in \mathcal{F}$. For more details on the RKHS, please see Wahba (1990); Steinwart and Christmann (2008); Schölkopf (2001) and references therein. The $\mathcal{M}_{q^{*}}$ is parametrized by parameters $\Pi_{q^{*}}^{*}=\left(\pi_{1}^{*}, \ldots, \pi_{q^{*}}^{*}\right) \in \Delta_{q^{*}-1}$ and $\Theta_{q^{*}}^{*}=\left(\beta_{1}^{*}, \ldots, \beta_{q^{*}}^{*}\right) \in \mathcal{F}^{q^{*}}$, where $\Delta_{q^{*}-1}$ is the $q^{*}$-simplex. We call $q^{*}$ the number of mixture components or the model order, $\Pi_{q^{*}}^{*}$ the mixing probabilities, and $\Theta_{q^{*}}^{*}$ the coefficient functions.

Suppose that we observe $n$ independently and identically distributed copies of $(Y, X)$, denoted as $\left\{\left(Y_{i}, X_{i}\right): i=1, \ldots, n\right\}$. Let $\mathcal{M}=\cup_{q} \mathcal{M}_{q}$. Let $f^{*}(y \mid x) \in \mathcal{M}_{q^{*}}$ be the true conditional distribution and $\widehat{f}(y \mid x) \in \mathcal{M}$ be the estimated conditional density. We are interested in investigating three 
important questions of interest for model (1.1):

(a) How to construct an estimator $\widehat{f}(y \mid x)$ that can achieve the optimal minimax rate of convergence?

(b) How to consistently estimate the number of mixture component $q^{*}$ ?

(c) How to numerically compute all unknown parameters and functions in $\widehat{f}(y \mid x)$ ?

It is well known that addressing these questions represents major challenges even for parametric mixture regression models (Chernoff, 1954, DacunhaCastelle and Gassiat, 1999; Zhu and Zhang, 2004; Ho and Nguyen, 2016, Heinrich and Kahn, 2018). The accuracy of estimation can be naturally measured by using

$$
\mathcal{R}_{n}\left(\widehat{f}, f^{*}\right)=\mathbb{E}_{X}\left\{\int\left(\sqrt{\widehat{f}(y \mid X)}-\sqrt{f^{*}(y \mid X)}\right)^{2} d y\right\}
$$

which is the squared Hellinger distance between $\widehat{h}(y, x)=\widehat{f}(y \mid x) f_{X}(x)$ and $h^{*}(y, x)=f^{*}(y \mid x) f_{X}(x)$. As the sample size $n$ increases, the convergence rate of $\mathcal{R}_{n}$ reflects the difficulty of the estimation problem.

We carry out a systematic investigation of model (1.1). We make four major contributions as follows:

(i) We construct an optimal estimate of $f^{*}(y \mid x)$ by using the following steps. We show that a minimax lower bound of $\mathcal{R}_{n}$ in $(1.2)$ depends 
on the reproducing kernel $K$, the covariance function $C$ of the random predictor $X$, and the model order $q^{*}$. In particular, it depends on the decay order of the eigenvalues of the operator $K^{1 / 2} C K^{1 / 2}$. Similar phenomenon has been found in the work on the functional regression (Cai and Yuan, 2012; Du and Wang, 2014; Wang and Ruppert, 2015). Then, we establish a minimax upper bound of $\mathcal{R}_{n}$ based on a penalized likelihood estimator when the true coefficient functions reside in $\mathcal{M}_{q}$ with $q \geq q^{*}$. We also propose a minimal penalty that yields a consistent order estimation.

(ii) We propose a general class of penalized likelihood order estimators in order to select and estimate the model order $q^{*}$. Theoretically, we establish the strong consistency of the order estimators for model (1.1). In contrast, most existing consistency results assume a prior upper bound on the order (Csiszár and Shields, 2000, Nishii, 1988). The only exception that we are aware is the reference Gassiat and Van Handel, 2012), which explores the consistency properties of penalized likelihood model order estimator and provides the minimal strongly consistency penalty.

(iii) We develop an Expectation-Maximization (EM) type algorithm to 
estimate all unknown parameters and functions.

(iv) We examine the finite sample performance of our methods by using simulations and a real data set collected by the Alzheimer's Disease Neuroimaging Initiative (ADNI) study.

The remainder of this paper is structured as follows. Section 2 establishes the minimax rate of convergence of $\mathcal{R}_{n}\left(\widehat{f}, f^{*}\right)$ in 1.2 . Section 3 presents the estimation of $q^{*}$ and the estimation of the coefficient functions $\Theta_{q^{*}}^{*}$. Section 4 summarizes the numerical results of all numerical experiments and real data analysis. This paper concludes with some discussions in Section 5.

\section{Methodology}

In this section, we first establish the minimax rate of convergence of $\mathcal{R}_{n}\left(\widehat{f}, f^{*}\right)$ in 1.2 and then introduce an estimation algorithm for $\widehat{f}$.

\subsection{Optimal Rate of Convergence}

The optimal rate of convergence of $\mathcal{R}_{n}\left(\widehat{f}, f^{*}\right)$ is established in several steps. We first derive a minimax lower bound and then show that the convergence rate of the lower bound is in fact optimal by constructing an estimator that can attain this rate of convergence. 


\subsection{Optimal Rate of Convergence}

\section{Minimax lower bound}

We first establish the minimax lower bound of $\mathcal{R}_{n}$ based on an assumption given below.

A1. Let $C(t, s)=\operatorname{cov}(X(t), X(s))$ be the covariance function of $X$ and $\left\{\rho_{k}: k \in \mathbb{N}\right\}$ be the non-increasing ordered eigenvalues of the operator $K^{1 / 2} C K^{1 / 2}$. Assume that $\rho_{k} \asymp k^{-2 r}$ with $r>0$.

Assumption A1 specifies that the decay rate of the eigenvalues of the operator $K^{1 / 2} C K^{1 / 2}$ is of order $k^{-2 r}$. For a concrete example, consider the univariate Sobolev space $W_{2}^{m}$. It is known that its reproducing kernel is $(m !)^{-2} B_{m}(s) B_{m}(t)+(-1)^{m-1}\{(2 m) !\}^{-1} B_{2 m}(|s-t|)$, where $B_{m}$ is the $m$ th Bernoulli polynomial (Wahba, 1990). It is known that the decay rate of the eigenvalues of this kernel is of order $k^{-2 m}$ (Micchelli and Wahba, 1981).

The minimax lower bound is given in the following theorem.

Theorem 1. Assuming A1 holds, we have

$$
\lim _{a \rightarrow 0} \lim _{n \rightarrow \infty} \inf _{\widehat{f}} \sup _{f^{*} \in \mathcal{M}_{q^{*}}} \mathbb{P}\left(\mathcal{R}_{n}\left(\widehat{f}, f^{*}\right) \geq a\left(q^{*} / n\right)^{\frac{2 r}{2 r+1}}\right)=1
$$

The result in Theorems 1 is an asymptotic result. It shows that, there exists a function $V_{1}(a)$ such that $\lim _{n \rightarrow \infty} \inf _{\widehat{f}} \sup _{f^{*}} \mathbb{P}\left(\mathcal{R}_{n}\left(\widehat{f}, f^{*}\right) \geq\right.$ $\left.a\left(q^{*} / n\right)^{2 r /(2 r+1)}\right) \geq V_{1}(a)$ and $V_{1}(a) \rightarrow 1$ as $a \rightarrow 0$. The proof of Theorem 
2.1 Optimal Rate of Convergence

1 is provided in the appendix. The main tool is adopted from Tsybakov (2009) by realizing that any lower bound for a specific case yields immediately a lower bound for the general case. Theorem 1 shows that the minimax lower bound depends on the model order $q^{*}$ and how the reproducing kernel $K$ and the covariance function $C$ are aligned. In general, the eigenvalues of $K$ and $C$ alone cannot determine the decay rate of the eigenvalues of $K^{1 / 2} C K^{1 / 2}$. For functional linear regression models, Cai and Yuan (2012) used the same tool to establish the minimax lower bound for prediction.

Minimax upper bound with $q \geq q^{*}$

Let $f^{*}(y \mid x) \in \mathcal{M}_{q^{*}}$ be the true conditional distribution and $\mathcal{M}=$ $\cup_{q=1}^{\infty} \mathcal{M}_{q}$. The $\log$-likelihood function can be written as

$$
\begin{aligned}
\ell_{n}(f) & =\sum_{i=1}^{n} \log f\left(Y_{i} \mid X_{i}\right) \\
& =\sum_{i=1}^{n} \log \sum_{k=1}^{q} \pi_{k} f_{0}\left(Y_{i}-\eta\left(X_{i}, \beta_{k}\right)\right), \quad f \in \mathcal{M},
\end{aligned}
$$

where $\eta(X, \beta)=\int_{\mathcal{I}} X(t) \beta(t) d t$. We consider a general class of penalized likelihood estimation given by

$$
\widehat{f}=\arg \min _{f \in \mathcal{M}_{q}}\left\{-\ell_{n}(f)+\lambda\|\beta\|_{K}^{2}\right\}
$$

for $q \geq q^{*}$, where $\lambda$ is a smoothing parameter that balances the trade-off between the goodness of fit to the data and the smoothness of the estimator and $\|\cdot\|_{K}$ is the RKHS norm. 
The following notations are used throughout:

$$
\begin{aligned}
& H_{0}(y \mid x)=\sup _{\eta \in \mathbb{R}} f_{0}(y-\eta) / f^{*}(y \mid x), H_{1}(y \mid x)=\sup _{\eta \in \mathbb{R}}\left|\dot{f}_{0}(y-\eta)\right| / f^{*}(y \mid x), \\
& H_{2}(y \mid x)=\sup _{\eta \in \mathbb{R}}\left|\ddot{f}_{0}(y-\eta)\right| / f^{*}(y \mid x), H_{3}(y \mid x)=\sup _{\eta \in \mathbb{R}}\left|f_{0}^{(3)}(y-\eta)\right| / f^{*}(y \mid x),
\end{aligned}
$$

where $\dot{f}_{0}, \ddot{f}_{0}$, and $f_{0}^{(3)}$ are the first-, second-, and third-order derivatives of $f_{0}(\cdot)$, respectively. We need two additional assumptions.

B1. $f_{0} \in C^{3}$ and $f_{0}(x)$ and $\dot{f}_{0}(x)$ vanish as $x \rightarrow \infty$. We assume $H_{k}(\cdot \mid \cdot) \in$ $L^{4}\left(h^{*} d \mu\right)$ for $k=0,1,2$ and $H_{3}(\cdot \mid \cdot) \in L^{2}\left(h^{*} d \mu\right)$.

B2. Given the functional predictor $X$, there exists a disjoint partition of $\mathbb{R}=A_{0} \cup\left\{\cup_{k=1}^{q^{*}} A_{k}\right\}$ such that $A_{1}, \ldots, A_{q^{*}}$ are bounded intervals, each bounded interval $A_{j}$ contains precisely one component $\eta_{k}^{*}=\left\langle X, \beta_{k}^{*}\right\rangle$ for $k=1, \ldots, q^{*}$, and the unbounded set $A_{0}$ contains no component.

Condition B1 is similar to the condition adopted in the reference (Gassiat and van Handel, 2014). This condition is satisfied in particular when $f_{0}$ is chosen as the standard normal density. Condition B2 characterizes the identifiability issue. In general, given $x$, the mean components $\left\langle x, \beta_{k}^{*}\right\rangle$ should be well separated to guarantee the identifiability.

Theorem 2. Assume that B1-B2 hold. Let $\left\{\rho_{k}: k \in \mathbb{N}\right\}$ be the nonincreasing ordered eigenvalues of the operator $K^{1 / 2} C K^{1 / 2}$. Assume that 
$\rho_{k} \asymp k^{-2 r}$ with $r>1 / 2$. For any $q \geq q^{*}$, we have

$$
\lim _{A \rightarrow \infty} \lim _{n \rightarrow \infty} \sup _{f^{*} \in \mathcal{M}_{q^{*}}} \mathbb{P}\left(\mathcal{R}_{n}\left(\widehat{f}, f^{*}\right) \leq A(q / n)^{\frac{2 r}{2 r+1}}\right)=1
$$

and $\|\widehat{\beta}\|_{K}^{2}=O_{p}(1)\left\|\beta^{*}\right\|_{K}^{2}$, provided that $\lambda$ is of order $q^{2 r /(2 r+1)} n^{1 /(2 r+1)}$.

The result in Theorem 2 is also an asymptotic result. It shows that there exists a function $V_{2}(a)$ such that $\lim _{n \rightarrow \infty} \inf _{\widehat{f}} \sup _{f^{*}} \mathbb{P}\left(\mathcal{R}_{n}\left(\widehat{f}, f^{*}\right) \leq\right.$ $\left.A\left(q^{*} / n\right)^{2 r /(2 r+1)}\right) \geq V_{2}(A)$ and $V_{2}(A) \rightarrow 1$ hold as $A \rightarrow \infty$. The proof of Theorem 2 is provided in the appendix. It combines two main tools. The first one is to characterize the local geometry of finite mixtures Gassiat and van Handel, 2014) and the second one is to use the empirical process theory with the covering numbers to establish the convergence rate van de Geer, 2000). Instead, the penalized estimator has an explicit solution for function linear regression models in Cai and Yuan (2012) and establishing the upper bound is more straightforward. Theorem 2 establishes the upper bound on the rate of the convergence of $\mathcal{R}_{n}$ based on the penalized likelihood estimator when $q \geq q^{*}$. However, we still cannot claim the minimax optimal rate of convergence since the model order $q^{*}$ is unknown. Therefore, the consistent estimation of $q^{*}$ is critical for achieving the minimax optimality on the rate of convergence of $\mathcal{R}_{n}$.

\section{Consistent order estimation}


The number of components $q^{*}$ of the true mixture $f^{*} \in \mathcal{M}$ can be estimated by using a general class of penalized likelihood order estimator:

$$
\widehat{q}_{n}=\arg \max _{q \geq 1}\left\{\sup _{f \in \mathcal{M}_{q}}\left(\ell_{n}(f)-\lambda\|\beta\|_{K}^{2}\right)-\operatorname{pen}_{n}(q)\right\}
$$

where $\operatorname{pen}_{n}(q)$ is a penalty function and $\ell_{n}(f)$ is the likelihood function. Our goal is to understand which $\operatorname{pen}_{n}(q)$ can yield the strong consistency of the order estimator, i.e., $\widehat{q}_{n} \rightarrow q^{*}$ as $n \rightarrow \infty$ a.s. Achieving this goal requires a precise understanding of the difference of the penalized log-likelihood functions given by

$$
\sup _{f \in \mathcal{M}_{q}}\left(\ell_{n}(f)-\lambda_{q, n}\|\beta\|_{K}^{2}\right)-\sup _{f \in \mathcal{M}_{q^{*}}}\left(\ell_{n}(f)-\lambda_{q^{*}, n}\|\beta\|_{K}^{2}\right)
$$

as $n \rightarrow \infty$, uniformly in the model order $q>q^{*}$. In this section, we discuss the selection of $\operatorname{pen}_{n}(q)$, which yields the strong consistency of $\widehat{q}_{n}$.

Theorem 3. Assume B1-B2 hold. Let $\left\{\rho_{k}: k \in \mathbb{N}\right\}$ be the nonincreasing ordered eigenvalues of the operator $K^{1 / 2} C K^{1 / 2}$. Assume that $\rho_{k} \asymp k^{-2 r}$ with $r>1 / 2$. Let $c>0$ be a constant and

$$
\operatorname{pen}_{n}(q)=c q^{\frac{2 r}{2 r+1}} n^{\frac{1}{2 r+1}} \log (n) .
$$

Then, we have

(a). $\widehat{q}_{n} \rightarrow q^{*}$ as $n \rightarrow \infty$ a.s.; 
(b). $\lim _{A \rightarrow \infty} \lim _{n \rightarrow \infty} \sup _{f^{*} \in \mathcal{M}_{q^{*}}} \mathbb{P}\left(\mathcal{R}_{n}\left(\widehat{f}, f^{*}\right) \geq A\left(q^{*} / n\right)^{\frac{2 r}{2 r+1}}\right)=0$.

The proof of Theorem 3 is provided in the appendix, which is obtained by precisely characterizing the difference of the penalized log-likelihood functions. The intuition for 2.8 is that, since we are dealing with infinite dimensional parameters like $\beta_{k}$, this order requires that the intrinsic dimension for each component is about $(n / q)^{1 /(2 r+1)}$ and the total dimension for $q$ components is $q^{2 r /(2 r+1)} n^{1 /(2 r+1)}$. Theorem 3 shows that the penalty term with the order $q^{\frac{2 r}{2 r+1}} n^{\frac{1}{2 r+1}} \log (n)$ yields a strong consistent order estimator. This term depends on the decay rate of the eigenvalues of the operator $K^{1 / 2} C K^{1 / 2}$. We may compare this with the popular BIC penalty

$$
\mathrm{BIC}_{n}(q)=\frac{d q+q-1}{2} \log (n)
$$

where $d$ is the dimension of the parameter space. However, due to the existence of the infinite dimensional parameters, it is not straightforward to choose the BIC penalty in our setting. Theorems $1-3$ together show that the minimax rate of convergence for $\mathcal{R}_{n}$ is $\left(q^{*} / n\right)^{\frac{2 r}{2 r+1}}$, which is determined by the rate of decay of the eigenvalues of the operator $K^{1 / 2} C K^{1 / 2}$ and the model order $q^{*}$. 


\subsection{The Estimation Algorithm}

The estimation procedure for model (1.1) consists of two parts: (i) the estimation of $q^{*}$ and (ii) the estimation of the coefficient functions $\Theta_{q^{*}}^{*}$. We propose a two-step estimation procedure as follows. First, for different values of $q$, we obtain the estimation of $\Theta_{q}$ for each given $q$. Second, based on the estimation of $\Theta_{q}$, we compute the term inside the maximum in (2.7) and then determine the estimate of $q^{*}$ accordingly. In this section, we fix $f_{0}$ to be a Gaussian density. By an abuse of notation, we use $f_{0}\left(Y_{i} \mid X_{i}, \beta_{k}, \sigma^{2}\right)$ to denote $f_{0}\left(Y_{i}-\left\langle X_{i}, \beta_{k}\right\rangle\right)$.

\section{EM algorithm}

For a given $q$, the estimate of $\Theta_{q}$ can be obtained via an ExpectationMaximization (EM) algorithm. Define $u_{i k}$ as the indicator of whether $X_{i}$ is from component $k$, i.e. $u_{i k}=1$ if $X_{i}$ comes from the $k$-th component, and $u_{i k}=0$ otherwise. If the missing data $u_{i k}$ 's could be observed, then the penalized log-likelihood for the complete data is given by

$\log L_{c}\left(\Theta_{q}, \sigma^{2}\right)=\sum_{i=1}^{n} \sum_{k=1}^{q} u_{i k}\left\{\log \pi_{k}+\log f_{0}\left(Y_{i} \mid X_{i}, \beta_{k}, \sigma^{2}\right)\right\}-\sum_{k=1}^{q} \frac{n \lambda\left\|\beta_{k}\right\|_{K}^{2}}{2 \sigma^{2}}$

where $\lambda$ controls the level of the penalty.

The corresponding EM algorithm is given in the following E-step and M-step: 


\section{E-step:}

Denote the estimates of parameters in the $m$-th iteration as $\Theta_{q}^{(m)}=$ $\left\{\beta_{1}^{(m)}, \ldots, \beta_{q}^{(m)}\right\}, \Pi_{q}^{(m)}=\left\{\pi_{1}^{(m)}, \ldots, \pi_{q}^{(m)}\right\}$, and $\sigma^{2(m)}$. We estimate $\tau_{i k}=$ $P\left(u_{i k}=1\right)$ as follows

$$
\tau_{i k}^{(m+1)}=\frac{\pi_{k}^{(m)} f_{0}\left(Y_{i} \mid X_{i}, \beta_{k}^{(m)}, \sigma^{2(m)}\right)}{\sum_{k=1}^{q} \pi_{k}^{(m)} f_{0}\left(Y_{i} \mid X_{i}, \beta_{k}^{(m)}, \sigma^{2(m)}\right)} .
$$

The mixing probabilities $\Pi^{(m+1)}$ are estimated accordingly by

$$
\pi_{k}^{(m+1)}=\frac{1}{n} \sum_{i=1}^{n} \tau_{i k}^{(m)}
$$

\section{M-step:}

We maximize the following $Q$ function with respect to $\Theta_{q}$ and $\sigma^{2}$ :

$$
\begin{aligned}
& Q\left(\Theta_{q}, \sigma^{2}\right) \\
= & \sum_{i=1}^{n} \sum_{k=1}^{q} \tau_{i k}^{(m)}\left(\log \pi_{k}+\log f_{0}\left(Y_{i} ; X_{i}, \beta_{k}, \sigma^{2}\right)\right)-\sum_{k=1}^{q} \frac{n \lambda\left\|\beta_{k}\right\|_{K}^{2}}{2 \sigma^{2}} .
\end{aligned}
$$

By the representer theorem (Wahba, 1990), for each $\beta_{k}$, there exists a vector $C_{k}=\left(c_{1 k}, \ldots, c_{n k}\right)^{T} \in R^{n}$ such that the quantity that maximizes the $Q$ function (2.11) can be expressed as:

$$
\beta_{k}(t)^{(m+1)}=\sum_{i=1}^{n} c_{i k} \int_{\mathcal{I}} K(t, s) X_{i}(s) d s
$$

and $\left\|\beta_{k}\right\|_{K}^{2}=C_{k}^{T} W C_{k}$, where $W=\left(W_{i j}\right)$ is an $n$ by $n$ Gram matrix with

$$
W_{i j}=\iint_{\mathcal{I} \times \mathcal{I}} X_{i}(s) K(s, t) X_{j}(t) d s d t \quad \text { for } i, j \in\{1, \ldots, n\} .
$$


Therefore, the maximization of the $Q$ function $(2.11$ can be solved componentwisely by solving the optimization with respect to $C_{k}$.

We consider the case when $f$ is the normal distribution, then the conditional distribution of $Y_{i}$, is given by

$$
Y_{i} \mid X_{i} \sim N\left(\int_{\mathcal{I}} X_{i}(t) \beta_{k}(t) d t, \sigma^{2}\right)=N\left(C_{k}^{T} W_{., i}, \sigma^{2}\right)
$$

where $W_{., i}$ is the $i$-th column of the Gram matrix 2.13).

The $Q$ function 2.11 then reduces to (up to a constant)

$$
\begin{aligned}
& Q\left(\Theta_{q}, \sigma^{2}\right) \\
= & \sum_{i=1}^{n} \sum_{k=1}^{q} \tau_{i k}^{(m)}\left(\log \pi_{k}-\frac{1}{2} \log \sigma^{2}-\frac{\left(Y_{i}-C_{k}^{T} W_{., i}\right)^{2}}{2 \sigma^{2}}\right)-\sum_{k=1}^{q} \frac{n \lambda C_{k}^{T} W C_{k}}{2 \sigma^{2}} .
\end{aligned}
$$

The estimate of $C_{k}$ in the above equation can be expressed as a Tikhonov regularized weighted least square problem. The solution is given by

$$
C_{k}^{(m+1)}=\left(W^{T} T_{k}^{(m)} W+n \lambda W\right)^{-1} W^{T} T_{k}^{(m)} Y
$$

where $T_{k}^{(m)}=\operatorname{diag}\left\{\tau_{1 k}^{(m)}, \ldots, \tau_{n k}^{(m)}\right\}$ and $Y=\left(Y_{1}, \ldots, Y_{n}\right)^{T}$.

We then construct the coefficient functions $\beta_{k}$ 's by plugging $c_{i k}^{(m+1)}$ s' into 2.12 , i.e.

$$
\beta_{k}(t)^{(m+1)}=\sum_{i=1}^{n} c_{i k}^{(m+1)} \int_{\mathcal{I}} K(t, s) X_{i}(s) d s .
$$


The estimation of $\sigma^{2}$ is achieved by solving the penalized MLE in 2.14, leading to

$$
\sigma^{2(m+1)}=\frac{\sum_{i=1}^{n} \sum_{k=1}^{q} \tau_{i k}^{(m)}\left(Y_{i}-C_{k}^{T(m+1)} W_{., i}\right)^{2}}{\sum_{i=1}^{n} \sum_{k=1}^{q} \tau_{i k}^{(m)}}+\frac{n \lambda \sum_{k=1}^{q} C_{k}^{T} W C_{k}}{\sum_{i=1}^{n} \sum_{k=1}^{q} \tau_{i k}^{(m)}} .
$$

We iteratively proceed the updates of (2.9) and 2.10) in E-step and the updates of 2.15), 2.16) and (2.17) in M-step until the convergence is reached.

We are using the Gaussian kernel throughout the paper. The bandwidth, denoted by $\varrho$, of the reproducing kernel $K(\cdot, \cdot)$ and the ridge penalty $\lambda$ are treated as hyper-parameters. They are tuned based on cross validation. Other kernels can be easily incorporated into our algorithm. Learning optimal kernels is definitely a non-trivial question and deserves further investigation. A good method of learning optimal kernels is to combine multiple candidate kernels.

\section{Order estimation}

The optimal order of the mixture model can be estimated through the

optimization in (2.7). This is a mixed integer programming (MIP), whose solution may not be directly obtained in practice. We denote the negative of Equation 2.7) inside the maximum as the order objective function, i.e.

$$
\operatorname{Order}(q)=\left(-\ell_{n}\left(f^{(q)}\right)+\lambda\left\|\beta^{(q)}\right\|_{K}^{2}\right)+\operatorname{pen}_{n}(q),
$$


where $f^{(q)}$ and $\beta^{(q)}$ are the estimates calculated from the EM algorithm with the model order being $q$. The first part of $\operatorname{Order}(q)$ is essentially the penalized loss and the second part is the penalty $(2.8)$ on $q$. Thus we conduct a sequential search for the optimal order $q^{*}$. Specifically, we start from $q=1$ and increase its value by 1 each time, and then compute the $\operatorname{Order}(q)$ according to 2.18 . As the model order increases, the penalized loss part in the $\operatorname{Order}(q)$ will decrease, and the penalty on the model order will increase. Once $\operatorname{Order}(q)$ no longer decreases, we select the $q$ value that minimizes $\operatorname{Order}(q)$ as the optimal estimate.

\section{Numerical Results}

We conduct simulations to evaluate the accuracy and efficiency of our methods and then apply them to the analysis of a real data set obtained from the ADNI study.

\subsection{Synthetic experiments}

We perform simulations in two different scenarios including one with two mixing components $\left(q^{*}=2\right)$ and another with three $\left(q^{*}=3\right)$. The true coefficient images are generated from a Gaussian function with different centers. The covariate images $X_{i}(t)$ 's are simulated from a Gaussian random 

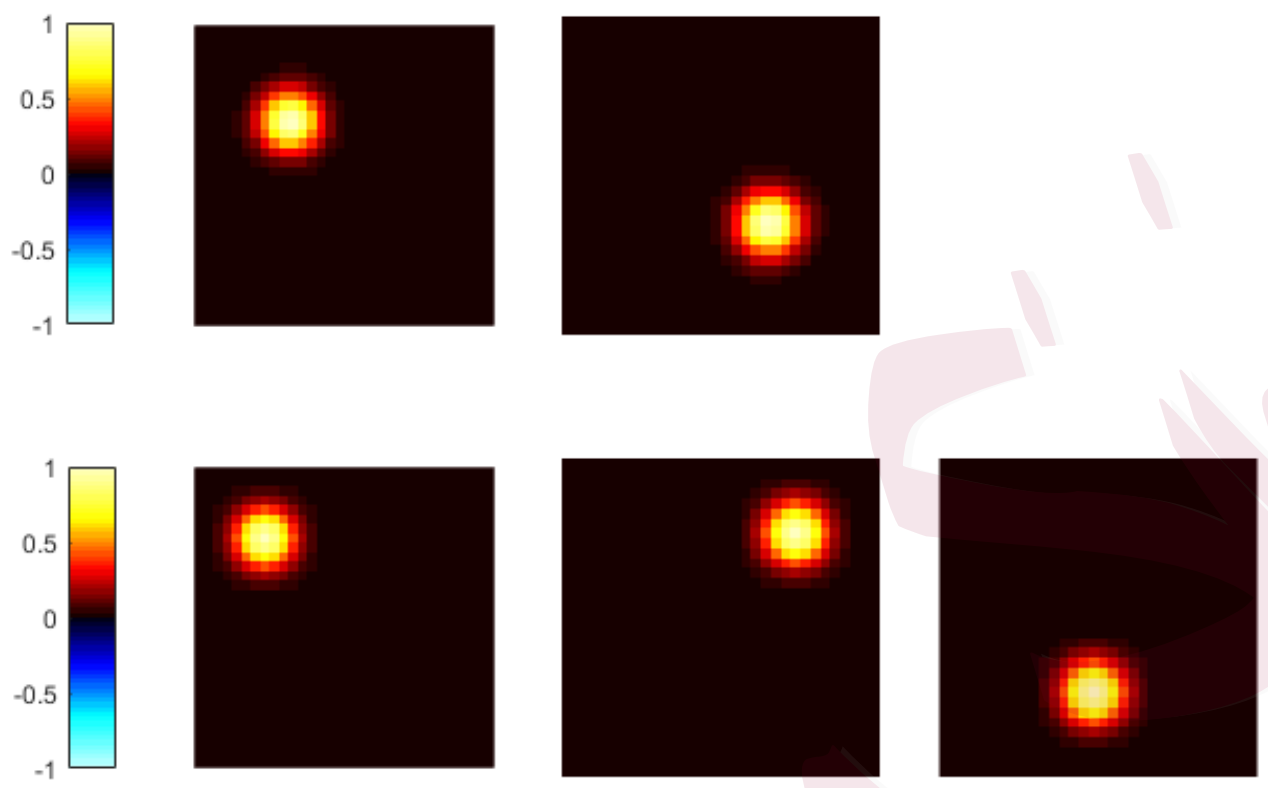

Figure 1: The first row of images includes the true coefficients $\beta_{1}$ and $\beta_{2}$ in the first scenario, and the bottom row includes the true coefficients $\beta_{1}, \beta_{2}$, and $\beta_{3}$ in the second scenario.

field. Figure 1 displays the true coefficients for these two scenarios.

The responses $Y_{i}$ 's follow a mixture of functional linear models given by

$$
Y_{i}=\sum_{k=1}^{q} \delta_{i k}\left(\beta_{k 0}+\int_{t \in \mathcal{T}} X_{i}(t) \beta_{k}(t) d t\right)+\epsilon_{i}
$$

where $\delta_{i k}=1$ if the subject $i$ comes from the $k$-th component, and 0 otherwise. The noise term $\epsilon_{i}$ follows a standard normal distribution and 

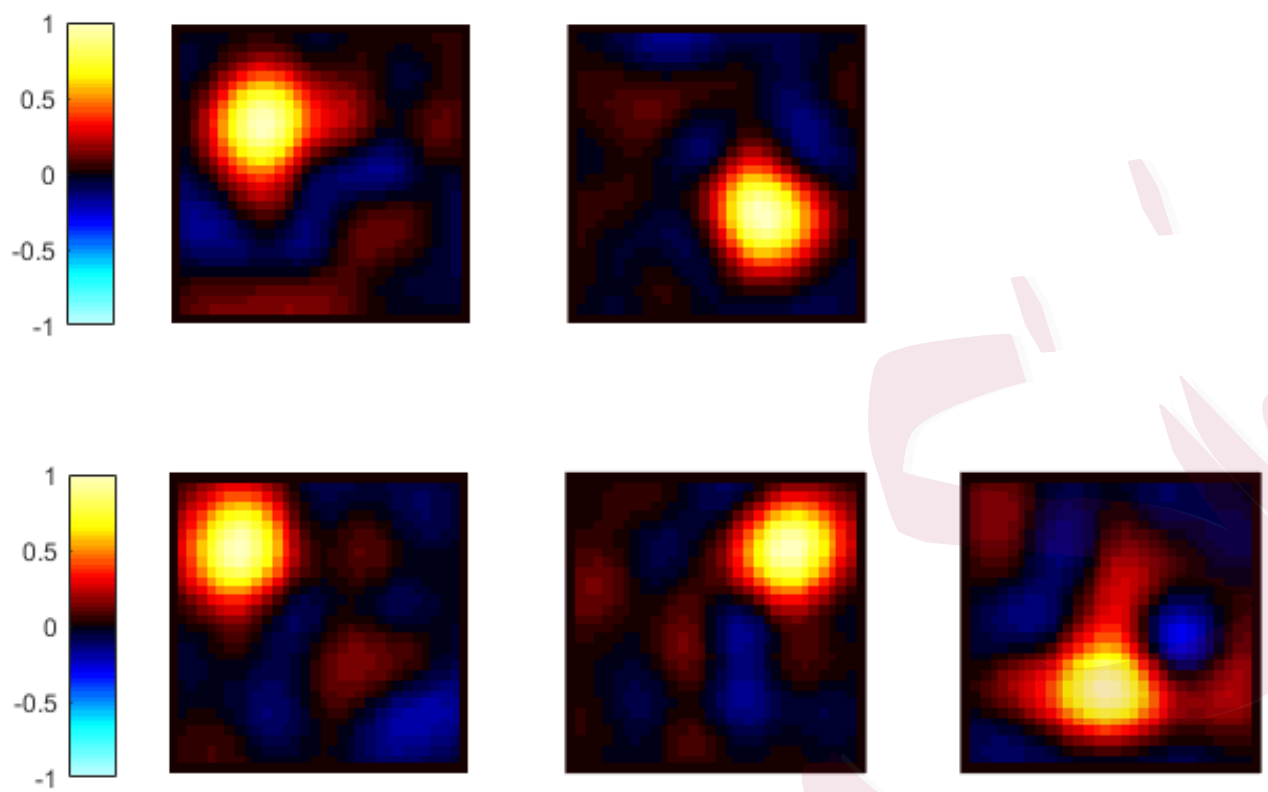

Figure 2: The top row of images includes the estimates of $\beta_{1}$ and $\beta_{2}$ in scenario 1 from one replication, and the bottom row is the estimates of $\beta_{1}$, $\beta_{2}$ and $\beta_{3}$ in scenarios 2 .

is independent of $X_{i}(t)$. In each scenario, we set the sample size in each components to be 80 , i.e., $\sum_{i=1}^{n} \delta_{i k}=80$, and the total number of samples are $n=160$ and 240 in scenarios 1 and 2 , respectively.

The initial tuning range for the bandwidth $\varrho$ of the kernel $K$ and the ridge penalty level $\lambda$ is from a 2-dimensional grid, i.e., $\sigma \otimes \lambda=\left\{2^{-5}, 2^{-4}, \cdots, 2^{5}\right\}^{\otimes 2}$. We start with the functional linear model with one component and increase 
the value $q$ by 1 each time. For each $q$, the stopping criteria of the EM algorithm is determined by either $\sum_{k=1}^{q}\left\|C_{k}^{(m+1)}-C_{k}^{(m)}\right\|^{2} /\left\{\sum_{k=1}^{q}\left\|C_{k}^{(m)}\right\|^{2}\right\} \leq$ $10^{-5}$ or the total number of iteration is over 500 . To evaluate the estimation performance, we first check the estimation of $q^{*}$. If $q^{*}$ is correctly estimated, we further compute the relative mean squared error (RMSE) for each $\beta_{k}$, i.e. $\mathrm{RMSE}=\left\|\widehat{\beta}_{k}-\beta_{k}\right\|^{2} /\left\|\beta_{k}\right\|^{2}$. For each sample, we denote $\widehat{u}_{i k}=1$, if $k=\arg \max _{j}\left\{\widehat{\tau}_{i j}\right\}$, and compute the assignment accuracy as ACC $=n^{-1} \sum_{i=1}^{n} \sum_{k=1}^{q^{*}} \delta\left(\widehat{u}_{i k}, u_{i k}\right)$, where $\delta(\cdot, \cdot)$ is the indicator function. The empirical prediction risk is quantified as the root mean square prediction error, i.e. $\mathrm{RMSPE}=\sqrt{\frac{1}{n} \sum_{i=1}^{n}\left(y_{i}-\widehat{y}_{i}\right)^{2}}$. We also conduct the regression analysis using just the functional linear model (FLM) (without the mixing components identification) as a baseline for the RMSPE comparison. Note that the basic FLM only has one component, so the RMSE and ACC comparisons are not applicable.

We present the estimation results in each scenario in Table 1. In both scenarios, we can correctly estimate the order of the mixing components $q^{*}$ in all 100 repetitions. In scenario 1 , the assignment accuracy is $86.5 \%$, whereas in scenario 2 , it is $65.1 \%$. This decrease of accuracy is mainly due to the increase of the number of components.

We achieve desirable relative mean square errors in both scenarios with 
3.2 MMSE score prediction in ADNI study

Table 1: The empirical mean and standard error in the parenthesis of relative mean squared error (RMSE), the assignment accuracy (ACC), and the root mean square prediction error (RMSPE) in scenarios 1 and 2, labeled as S1 and S2, respectively. FFMRM denotes the functional finite mixture regression model; FLM denotes the functional linear model. For each case, 100 simulated data sets were used.

\begin{tabular}{|c|c|c|c|c|c|c|}
\hline & & RMSE & & $\mathrm{ACC}$ & RM & SPE \\
\hline & $\beta_{1}$ & $\beta_{2}$ & $\beta_{3}$ & & FFMRM & FLM \\
\hline S1 & $1.80(0.38)$ & $1.84(0.39)$ & $\mathrm{N} / \mathrm{A}$ & $0.86(0.06)$ & $1.01(0.47)$ & $3.65(0.35)$ \\
\hline $\mathrm{S} 2$ & $1.99(0.39)$ & $2.04(0.37)$ & $2.10(0.28)$ & $0.65(0.23)$ & $1.02(0.34)$ & $3.53(0.32)$ \\
\hline
\end{tabular}

a slightly better performance in scenario 1 . To graphically demonstrate the estimated coefficient, we randomly pick up one iteration for each scenario and plot the estimated coefficients in Figure 2, The estimated coefficients can correctly capture the non-zeros regions and recover the patterns in the true coefficients.

\subsection{MMSE score prediction in ADNI study}

To further demonstrate the usefulness of model $(1.1)$, we apply our methods to a real data set obtained from the Alzheimer's Disease Neuroimaging 
3.2 MMSE score prediction in ADNI study

Table 2: The demographical information of all participants in ADNI. The means are reported with the standard deviations included in the parentheses.

\begin{tabular}{lccc}
\hline Diagnosis & Gender(F/M) & Age & MMSE \\
& \multicolumn{3}{c}{ (years) } \\
\hline CN & $164 / 167$ & $74.46(5.58)$ & $28.99(1.23)$ \\
SMC & $61 / 42$ & $72.01(5.46)$ & $29.01(1.96)$ \\
EMCI & $127 / 161$ & $71.26(7.51)$ & $28.35(1.55)$ \\
LMCI & $152 / 245$ & $73.66(7.41)$ & $26.97(2.70)$ \\
AD & $99 / 142$ & $74.96(7.88)$ & $23.25(2.10)$ \\
\hline
\end{tabular}

Initiative (ADNI) study. The ADNI study as a large scale multi-site study has collected g magnetic resonance imaging (MRI), positron emission tomography (PET) images, Cerebrospinal fluid (CSF), and blood biomarkers, among many others. More information about this study can be found at the ADNI website (http://adni.loni.usc.edu/).

We study the Positron Emission Tomography (PET) data, and use them as imaging predictors to predict the Mini-Mental State Examination (MMSE) scores. The PET images measure the metabolic processes of the patients, such as flows of blood to different parts of the brain, via detect- 
3.2 MMSE score prediction in ADNI study

ing the radioactivity of the injected tracer. We analyze the PET images collected at the baseline of the ANDI study. Among a total number of 1,360 participants, there are five diagnostic groups, including Normal Control (CN), Significant Memory Concern (SMC), Early Mild Cognitive Impairment (EMCI), Late Mild Cognitive Impairment (LMCI) and diagnosed Alzheimer's Disease (AD). The demographical information of the participants is summarized in Table 2 and a sample image from each type of participants is provided in Figure 3 .

We remove the age and gender factors by fitting a linear model in each voxel and use the residual maps as the covariate images of model (1.1). The same configuration of the EM algorithm in the simulation studies is used to train the model and the adaptive tuning procedure is utilized for tuning parameter selection. Finally, three mixing components are determined by the EM algorithm with their corresponding coefficients images displayed in Figure 4. The overall proportional probability $\pi_{k}$ 's are estimated as $19.8 \%$, $41.0 \%$, and $39.2 \%$, respectively. We conduct a hierarchical clustering on the patients according to their estimated proportional probabilities to each component $\tau_{i k}$ and illustrate the corresponding similarity matrix in Figure 5. These clusters indicate potential heterogeneous disease patterns of $\mathrm{AD}$ among the population, which is a fact that has been proved by existing 


\subsection{MMSE score prediction in ADNI study}

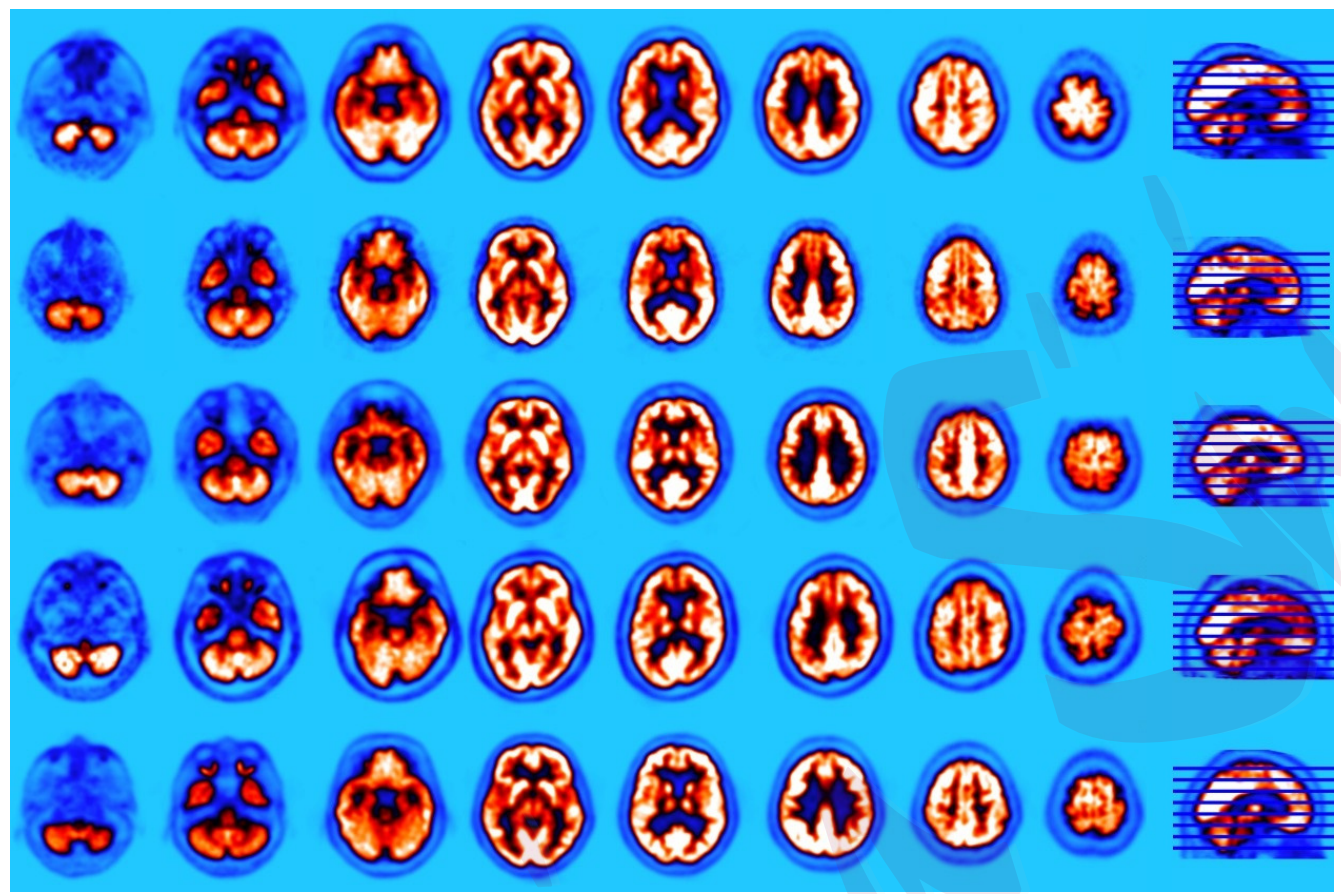

Figure 3: The five rows of images are the PET images of different types of participants. We randomly pick one image from each group for illustration. The types of the participants are AD, CN, EMCI, LMCI and SMC, from top to bottom respectively. Eight transverse slices located at $Z=8$ to 78 with equal increment of 10, are displayed from left to right, respectively.

studies (Latta et al., 2015; Dong et al., 2016). We overlay the coefficient images on the MNI-152 ROI template (Fonov et al., 2011), and are able to identify several regions of interest: $\widehat{\beta}_{1}$ mainly represents the impairment in sup frontal and parietal lobe; $\widehat{\beta}_{2}$ highlights corpus callosum and occipital 


\subsection{MMSE score prediction in ADNI study}

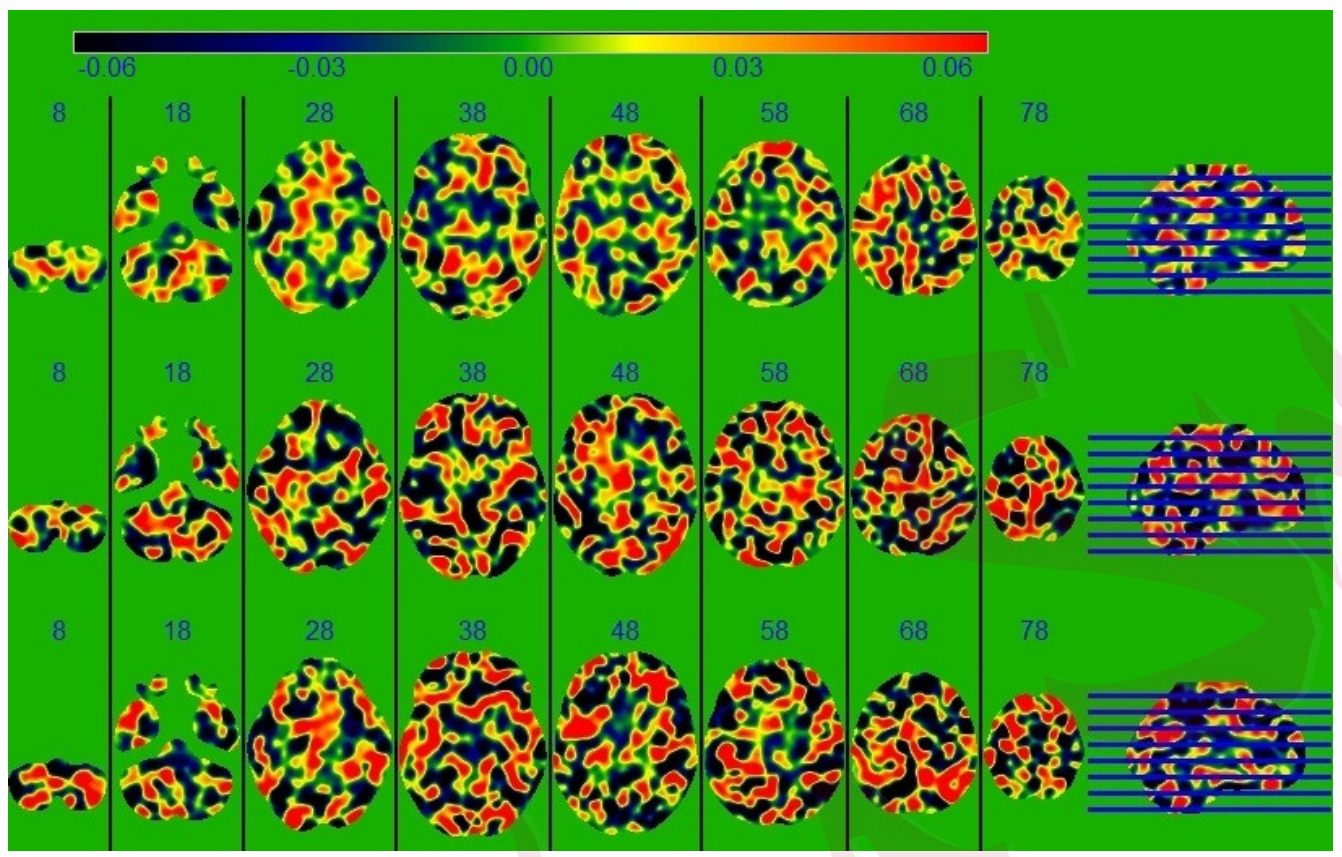

Figure 4: The three rows of images are the estimated coefficients, $\widehat{\beta}_{1}, \widehat{\beta}_{2}$ and $\widehat{\beta}_{3}$ from top to bottom, respectively. Eight transverse slices located at $Z=8$ to 78 with equal increment of 10 , are displayed from left to right, respectively.

lobe; and $\widehat{\beta}_{3}$ mostly covers the middle frontal gyrus. Many studies in literature have shown that the reduced glucose metabolic activities and structural impairment in frontal lobe, parietal lobe and occipital lobe of the cerebrum are associated with the degradation in cognition and the progression to Alzheimer's Disease (Lin et al., 2017; Heneka et al., 2015). 


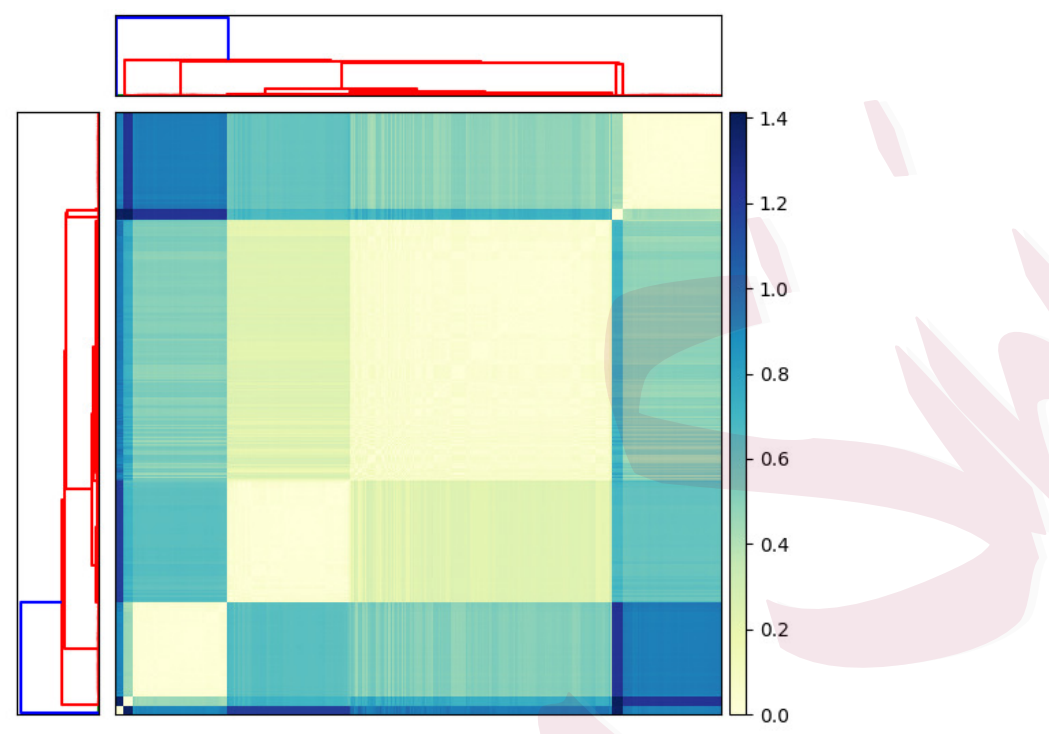

Figure 5: The similarity matrix of the estimation of $\tau_{k}$ 's. The value indicates the pairwise Euclidean distance of the proportional probabilities.

\section{Conclusion}

We have developed a functional finite mixture regression model with functional predictors in the reproducing kernel Hilbert space. This is a challenging problem because of the unknown model order and the existence of infinite dimensional unknown parameters. Our theoretical developments discover a strong consistent estimator of the model order using a penalized likelihood estimation and establish the minimax rate of convergence 


\section{REFERENCES}

for the estimation risk. It is shown that the optimal rate is jointly determined by the alignment of the reproducing kernel, covariance kernel, and the model order. An efficient EM algorithm is also proposed, and empirical experimental results demonstrate the merits of our method.

\section{References}

Bowman, A. (2010). Functional Data Analysis with $R$ and MATLAB, Volume 34. Foundation for Open Access Statistics.

Cai, T. and P. Hall (2006). Prediction in functional linear regression. The Annals of Statistics, 34(5), 2159-2179.

Cai, T. T. and M. Yuan (2012). Minimax and adaptive prediction for functional linear regression. Journal of the American Statistical Association 107(499), 1201-1216.

Calinon, S., G. F., and A. Billard (2007). On learning, representing, and generalizing a task in a humanoid robot. IEEE Transactions on Systems, Man, and Cybernetics, Part B (Cybernetics) 37, 286-298.

Chernoff, H. (1954). On the distribution of the likelihood ratio. The Annals of Mathematical Statistics 25, 573-578.

Crambes, C., A. Kneip, and P. Sarda (2009). Smoothing splines estimators for functional linear regression. The Annals of Statistics 37(1), 35-72.

Csiszár, I. and P. C. Shields (2000). The consistency of the bic markov order estimator. The 


\section{REFERENCES}

Annals of Statistics 28(6), 1601-1619.

Dacunha-Castelle, D. and E. Gassiat (1999). Testing the order of a model using locally conic parametrization: population mixtures and stationary arma processes. The Annals of Statistics $27(4), 1178-1209$.

Dong, A., J. B. Toledo, N. Honnorat, J. Doshi, E. Varol, A. Sotiras, D. Wolk, J. Q. Trojanowski, C. Davatzikos, and A. D. N. Initiative (2016). Heterogeneity of neuroanatomical patterns in prodromal alzheimer's disease: links to cognition, progression and biomarkers. Brain 140(3), 735-747.

Du, P. and X. Wang (2014). Penalized likelihood functional regression. Statistica Sinica 24, 1017-1041.

Feng, X., T. Li, X. Song, and H. Zhu (2020). Bayesian scalar on image regression with nonignorable nonresponse. Journal of the American Statistical Association 115(532), 1574-1597.

Ferraty, F. and P. Vieu (2006). Nonparametric Functional Data Analysis. Springer Series in Statistics. Springer Science, New York, NY.

Fonov, V., A. C. Evans, K. Botteron, C. R. Almli, R. C. McKinstry, D. L. Collins, and B. D. C. Group (2011). Unbiased average age-appropriate atlases for pediatric studies. Neuroimage $54(1), 313-327$.

Gassiat, E. and R. Van Handel (2012). Consistent order estimation and minimal penalties. IEEE Transactions on Information Theory 59(2), 1115-1128. 


\section{REFERENCES}

Gassiat, E. and R. van Handel (2014). The local geometry of finite mixtures. Transactions of the American Mathematical Society 366, 1047 - 1072.

Ghahramani, Z. and M. I. Jordan (1994). Supervised learning from incomplete data via an em approach. Advances in Neural Information Processing Systems 6, 120-127.

Hall, P. and J. L. Horowitz (2007). Methodology and convergence rates for functional linear regression. The Annals of Statistics 35(1), 70-91.

Heinrich, P. and J. Kahn (2018). Strong identifiability and optimal minimax rates for finite mixture estimation. The Annals of Statistics 46(6A), 2844-2870.

Heneka, M. T., M. J. Carson, J. El Khoury, G. E. Landreth, F. Brosseron, D. L. Feinstein, A. H. Jacobs, T. Wyss-Coray, J. Vitorica, and R. M. Ransohoff (2015). Neuroinflammation in alzheimer's disease. The Lancet Neurology 14(4), 388-405.

Ho, N. and X. Nguyen (2016). Convergence rates of parameter estimation for some weakly identifiable finite mixtures. Annals of Statistics 44(6), 2726-2755.

Latta, C. H., H. M. Brothers, and D. M. Wilcock (2015). Neuroinflammation in alzheimer's disease; a source of heterogeneity and target for personalized therapy. Neuroscience 302, 103-111.

Lin, F., P. Ren, R. Y. Lo, B. P. Chapman, A. Jacobs, T. M. Baran, A. P. Porsteinsson, and J. J. Foxe (2017). Insula and inferior frontal gyrus' activities protect memory performance against alzheimer's disease pathology in old age. Journal of Alzheimer's Disease 55(2), 669-678. 


\section{REFERENCES}

Liu, R. and H. Zhu (2021). Statistical disease mapping for heterogeneous neuroimaging studies. Canadian Journal of Statistics 49(1), 10-34.

McLachlan, G. and D. Peel (2000). Finite Mixture Models. New York: Wiley.

Micchelli, C. and G. Wahba (1981). Design problems for optimal surface interpolation. In Z. Ziegler (Ed.), Approximation Theory and Applications, pp. 329-347. Academic Press, New York.

Nishii, R. (1988). Maximum likelihood principle and model selection when the true model is unspecified. Journal of Multivariate Analysis 27(2), 392-403.

Ombao, H., M. Lindquist, W. Thompson, and J. Aston (2016). Handbook of neuroimaging data analysis. CRC Press.

Ramsay, J. and B. Sloverman (2005). Functional Data Analysis. Springer Series in Statistics. Springer Science, New York, NY.

Richard, S. and P. Green (1997). On bayesian analysis of mixtures with an unknown number of components. Journal of Royal Statistical Society B 59, 731-792.

Schölkopf, B. (2001). The kernel trick for distances. In T. K. Leen, T. G. Dietterich, and V. Tresp (Eds.), Advances in Neural Information Processing Systems 13, pp. 301-307. MIT Press.

Steinwart, I. and A. Christmann (2008). Support Vector Machines. Springer Science \& Business Media. 


\section{REFERENCES}

Stulp, F. and O. Sigaud (2015). Many regression algorithms, one unified model: A review. Neural Networks 69, 60-79.

Tsybakov, A. (2009). Introduction to Nonparametric Estimation. Springer, New York. van de Geer, S. A. (2000). Applications of Empirical Process Theory. Cambridge, U.K.: Cambridge University Press.

van de Geer, S. A. (2001). Empirical Processes in M-Estimation. Cambridge, U.K.: Cambridge University Press.

Wahba, G. (1990). Spline Models for Observational Data, Volume 59. Siam.

Wang, J. L., J. M. Chiou, and H. G. Muller (2016). Review of functional data analysis. Annual Review of Statistics and Its Application 3, 257-295.

Wang, X. and D. Ruppert (2015). Optimal prediction in an additive functional model. Statistica Sinica 25, 567-589.

Wang, X., H. Zhu, and ADNI (2017). Generalized scalar-on-image regression models via total variation. Journal of the American Statistical Association 112(519), 1156-1168.

Wang, Y. R., L. Li, J. J. Li, and H. Huang (2021). Network modeling in biology: statistical methods for gene and brain networks. Statistical Science 36(1), 89-108.

Yao, F., Y. Fu, and T. Lee (2011). Functional mixture regression. Biostatistics 12(2), 341-353.

Yuan, M. and T. T. Cai (2010). A reproducing kernel hilbert space approach to functional linear regression. The Annals of Statistics 38(6), 3412-3444. 
Zhu, H.-T. and H. Zhang (2004). Hypothesis testing in mixture regression models. Journal of the Royal Statistical Society: Series B 66(1), 3-16.

Department of Statistics, Purdue University, Indiana, U.S.A.

E-mail: wangxiao@purdue.edu

Department of Statistics and Operations Research, University of North Carolina at Chapel Hill,

North Carolina, U.S.A.

E-mail: leo1986@unc.edu

Department of Biostatistics, University of North Carolina at Chapel Hill, North Carolina, U.S.A.

E-mail: htzhu@email.unc.edu 


\section{Appendix: Technical Lemmas and Proofs of Theorems}

\section{A1. Technical Lemmas}

Lemma 1. Assume B1 and B2 hold. Define $H_{q}(\epsilon)=\left\{\sqrt{f / f^{*}}: f \in \mathcal{M}_{q}, \mathcal{H}\left(h, h^{*}\right) \leq \epsilon\right\}$. Then

$$
N_{[]}\left(H_{q}(\epsilon), \delta\right) \leq\left(\frac{C_{1} \epsilon}{\delta}\right)^{10\left(C_{2} \delta^{-\frac{1}{r}}+1\right) q+1}
$$

for all $q \geq q^{*}$ and $\delta / \epsilon \leq 1$.

Proof. We prove the lemma by extending the arguments in Gassiat and van Handel (2014).

Note that $f / f^{*}=h / h^{*}$. We denote by $\|\cdot\|_{p}$ the $L^{p}\left(h^{*} d \mu\right)$-norm, i.e., $\|g\|_{p}^{0}=\int|g|^{p} h^{*} d \mu$.

Denote $\eta_{i}^{*}=\left\langle x, \beta_{i}^{*}\right\rangle$ and $\eta_{j}=\left\langle x, \beta_{j}\right\rangle, i=1, \ldots, q^{*}, j=1, \ldots, q$. It follows from B2 that we can find a partition of $\mathbb{R}, A_{0}, A_{1}, \ldots, A_{q^{*}}$, such that each bounded set $A_{i}, i=1, \ldots, q^{*}$, contains precisely one component $\eta_{i}^{*}=\left\langle X, \beta_{i}^{*}\right\rangle$ and the unbounded set $A_{0}=\mathbb{R}^{M} \backslash\left(A_{1} \cup \cdots \cup A_{q^{*}}\right)$ contains no component. Let $f \in \mathcal{M}_{q}$, so that we can write $f=\sum_{i=1}^{q} \pi_{i} f_{0}\left(y-\eta_{i}\right)$. Then,

$$
\begin{aligned}
\frac{f-f^{*}}{f^{*}}= & \sum_{j: \eta_{j} \in A_{0}} \pi_{j} \frac{f_{0}\left(y-\eta_{j}\right)}{f^{*}} \\
& +\sum_{i=1}^{q^{*}}\left\{\left(\sum_{j: \eta_{j} \in A_{i}} \pi_{j}-\pi_{i}^{*}\right) \frac{f_{0}\left(y-\eta_{i}^{*}\right)}{f^{*}}+\sum_{j: \eta_{j} \in A_{i}} \frac{f_{0}\left(y-\eta_{j}\right)-f_{0}\left(y-\eta_{i}^{*}\right)}{f^{*}}\right\} .
\end{aligned}
$$

Taylor expansion gives

$$
f_{0}\left(y-\eta_{j}\right)-f_{0}\left(y-\eta_{i}^{*}\right)=\dot{f}_{0}\left(y-\eta_{i}^{*}\right)\left(\eta_{j}-\eta_{i}^{*}\right)+\frac{1}{2} \ddot{f}_{0}(y-\tilde{\eta})\left(\eta_{j}-\eta_{i}^{*}\right)^{2}
$$


Using Assumption B2, we find that

$$
\begin{aligned}
\left|\frac{f-f^{*}}{f^{*}}\right| \leq\left[\sum_{j: \eta_{j} \in A_{0}} \pi_{j}\right. & +\sum_{i=1}^{q^{*}}\left\{\left|\sum_{j: \eta_{j} \in A_{i}} \pi_{j}-\pi_{i}^{*}\right|+\left|\sum_{j: \eta_{j} \in A_{i}} \pi_{j}\left(\eta_{j}-\eta_{i}^{*}\right)\right|\right. \\
& \left.\left.+\frac{1}{2} \sum_{j: \eta_{j} \in A_{i}} \pi_{j}\left(\eta_{j}-\eta_{i}^{*}\right)^{2}\right\}\right]\left(H_{0}+H_{1}+H_{2}\right) .
\end{aligned}
$$

On the other hand, it follows from Theorem 3.10 of Gassiat and van Handel (2014) that there

exists a constant $c^{*}$ such that

$$
\begin{aligned}
\left\|\frac{f-f^{*}}{f^{*}}\right\|_{1} \geq c^{*}\left[\sum_{j: \eta_{j} \in A_{0}} \pi_{j}\right. & +\sum_{i=1}^{q^{*}}\left\{\left|\sum_{j: \eta_{j} \in A_{i}} \pi_{j}-\pi_{i}^{*}\right|+\left|\sum_{j: \eta_{j} \in A_{i}} \pi_{j}\left(\eta_{j}-\eta_{i}^{*}\right)\right|\right. \\
& \left.\left.+\frac{1}{2} \sum_{j: \eta_{j} \in A_{i}} \pi_{j}\left(\eta_{j}-\eta_{i}^{*}\right)^{2}\right\}\right] .
\end{aligned}
$$

Hence, for all $f \in \mathcal{M}$,

$$
\frac{\left|f / f^{*}-1\right|}{\left\|f / f^{*}-1\right\|_{1}} \leq S:=\frac{1}{c^{*}}\left(H_{0}+H_{1}+H_{2}\right)
$$

In addition, using $|\sqrt{x}-1| \leq|x-1|$, we find

$$
\frac{\left|\sqrt{h / h^{*}}-1\right|}{\mathcal{H}\left(h, h^{*}\right)} \leq \frac{\left|h / h^{*}-1\right|}{\frac{1}{2}\left\|h / h^{*}-1\right\|_{1}}=\frac{\left|f / f^{*}-1\right|}{\frac{1}{2}\left\|f / f^{*}-1\right\|_{1}} \leq 2 S .
$$

Similar to Lemma 3.15 of [1], for and $f \in \mathcal{M}$, we have

$$
\frac{\left|\sqrt{h / h^{*}}-1\right|}{H\left(h, h^{*}\right)}-\frac{h / h^{*}-1}{\sqrt{\chi^{2}\left(h \| h^{*}\right)}} \leq\left(4\|S\|_{4}^{2} S+2 S^{2}\right) H\left(h, h^{*}\right),
$$

where the chi-square divergence is defined as $\chi^{2}\left(h \| h^{*}\right)=\int\left(h / h^{*}-1\right)^{2} h^{*} d \mu$. This allows us to make further approximation based on Lemma 3.16 of [1]. Let $\alpha>0$, and for every $f \in \mathcal{M}_{q}$ such that $H\left(h, h^{*}\right) \leq \alpha$. Define

$$
\tilde{\ell}=\sum_{i=1}^{q^{*}}\left\{a_{i} \frac{f_{0}\left(y-\eta_{i}^{*}\right)}{f^{*}}+b_{i} \frac{\dot{f}_{0}\left(y-\eta_{i}^{*}\right)}{f^{*}}+e_{i}^{2} \frac{\ddot{f}_{0}\left(y-\eta_{i}^{*}\right)}{f^{*}}\right\}+\sum_{j=1}^{q} \gamma_{j} \frac{f_{0}\left(y-\eta_{j}\right)}{f^{*}}
$$




\section{REFERENCES}

where

$$
\begin{aligned}
& \sum_{i=1}^{q^{*}}\left|a_{i}\right| \leq \frac{1}{c^{*}}+\frac{1}{\sqrt{c^{*} \alpha}}, \quad \sum_{i=1}^{q^{*}}\left|b_{i}\right| \leq \frac{1}{c^{*}}+2 \frac{c}{\sqrt{c^{*} \alpha}} \\
& \sum_{i=1}^{q^{*}} e_{i}^{2} \leq \frac{1}{c^{*}}, \quad \sum_{j=1}^{q} \gamma_{j} \leq \frac{1}{\sqrt{c^{*} \alpha} \wedge c^{*}}
\end{aligned}
$$

We have

$$
\left|\frac{h / h^{*}-1}{\sqrt{\chi^{2}\left(h \| h^{*}\right)}}-\tilde{\ell}\right| \leq \frac{\sqrt{2}}{2\left(c^{*}\right)^{5 / 4}}\left(\left\|H_{3}\right\|_{2} S+H_{3}\right) \alpha^{1 / 4}
$$

Define

$$
d_{f}=\frac{\sqrt{f / f^{*}}-1}{\left\|\sqrt{f / f^{*}}-1\right\|_{2}}
$$

and

$$
\mathcal{D}_{q}=\left\{d_{f}: f \in \mathcal{M}_{q}, f \neq f^{*}\right\}, \quad \mathcal{D}_{q, \alpha}=\left\{d_{f}: f \in \mathcal{M}_{q}, f \neq f^{*}, \mathcal{H}\left(h, h^{*}\right) \leq \alpha\right\}
$$

Then,

$$
N_{[]}\left(\mathcal{D}_{q}, \delta\right) \leq N_{[]}\left(\mathcal{D}_{q, \alpha}, \delta\right)+N_{[]}\left(\mathcal{D}_{q} \backslash \mathcal{D}_{q, \alpha}, \delta\right)
$$

We estimate both bracketing numbers separately. Define a family of functions

$$
\tilde{\mathcal{L}}_{q, \alpha}=\left\{\sum_{i=1}^{q^{*}}\left\{a_{i} \frac{f_{0}\left(y-\eta_{i}^{*}\right)}{f^{*}}+b_{i} \frac{\dot{f}_{0}\left(y-\eta_{i}^{*}\right)}{f^{*}}+e_{i}^{2} \frac{\ddot{f}_{0}\left(y-\eta_{i}^{*}\right)}{f^{*}}\right\}+\sum_{j=1}^{q} \gamma_{j} \frac{f_{0}\left(y-\eta_{j}\right)}{f^{*}}\right\}
$$

where $(a, b, e, \gamma) \in \mathbb{R}^{q^{*}} \times R^{q^{*}} \times R^{q^{*}} \times R^{q}$ satisfies the constraint 4.21. For $\tilde{l}, \tilde{l}^{\prime} \in \tilde{\mathcal{L}}_{q, \alpha}$,

$$
\begin{aligned}
\left|\tilde{l}-\tilde{l}^{\prime}\right| \leq & H_{0} \sum_{i=1}^{q^{*}}\left|a_{i}-a_{i}^{\prime}\right|+H_{1} \sum_{i=1}^{q^{*}}\left|b_{i}-b_{i}^{\prime}\right|+H_{0} \sum_{i=1}^{q}\left|\gamma_{i}-\gamma_{i}^{\prime}\right| \\
& +\frac{1}{\sqrt{c^{*} \alpha} \wedge c^{*}} H_{1} \sum_{i=1}^{q}\left|\left\langle x, \beta_{i}-\beta_{i}^{\prime}\right\rangle\right|+\frac{2 H_{2}}{\sqrt{c^{*}}} \sum_{i=1}^{q^{*}}\left|e_{i}-e_{i}^{\prime}\right| .
\end{aligned}
$$




\section{REFERENCES}

Note that

$$
\begin{aligned}
\mathbb{E}_{X}\left(\sum_{i=1}^{q}\left|\left\langle x, \beta_{i}-\beta_{i}^{\prime}\right\rangle\right|\right)^{2} & \leq q \sum_{i=1}^{q} \mathbb{E}_{X}\left|\left\langle x, \beta_{i}-\beta_{i}^{\prime}\right\rangle\right|^{2}=q \sum_{i=1}^{q} \sum_{k=1}^{\infty} \rho_{k}\left(g_{i k}-g_{i k}^{\prime}\right)^{2} \\
& \leq q \sum_{i=1}^{q} \sum_{k=1}^{M} \rho_{k}\left(g_{i k}-g_{i k}^{\prime}\right)^{2}+M^{-2 r} o_{M}(1) .
\end{aligned}
$$

We may choose $M=c_{1} \delta^{-1 / r}$, so that

$$
\left|\tilde{l}-\tilde{l}^{\prime}\right| \leq V\left\|(a, b, \gamma, g, e)-\left(a^{\prime}, b^{\prime}, \gamma^{\prime}, g^{\prime}, e^{\prime}\right)\right\| \operatorname{norm}+o(\delta)
$$

where $V=3\left(H_{0}+H_{1}+H_{2}\right)$ and $\|\cdot\|$ norm is the norm on $\mathbb{R}^{3 q^{*}+c_{2} q\left(\delta^{-1 / r}+1\right)}$ defined by

$$
\|(a, b, \gamma, g, e)\|_{\text {norm }}=\sum_{i=1}^{q^{*}}\left|a_{i}\right|+\sum_{i=1}^{q^{*}}\left|b_{i}\right|+\sum_{i=1}^{q}\left|\gamma_{i}\right|+\frac{q}{\sqrt{c^{*} \alpha} \wedge c^{*}} \sum_{i=1}^{q} \sum_{k=1}^{M} \rho_{k} g_{i k}^{2}+\frac{2}{\sqrt{c^{*}}} \sum_{i=1}^{q^{*}}\left|e_{i}\right| .
$$

Using the standard fact of the covering number for the Euclidean ball we obtain

$$
N_{[]}\left(\tilde{L}_{q, \alpha}, \delta\right)=\left(\frac{c_{3}+\delta}{\delta}\right)^{3 q^{*}+c_{2} q\left(\delta^{-1 / r}+1\right)}
$$

Since $q \geq q^{*}$ and $\delta \leq 1$, we therefore obtain

$$
N_{[]}\left(\mathcal{D}_{q, \alpha}, \delta\right) \leq\left(\frac{c_{4}}{\delta}\right)^{c_{5} q\left(\delta^{-1 / r}+1\right)}
$$

Lemma 2. Assume B1 and B2 hold.

(a).

$$
\mathbb{P}\left[\sup _{f \in \mathcal{M}_{q}} \sum_{i=1}^{n} \log \frac{f\left(Y_{i} \mid X_{i}\right)}{f^{*}\left(Y_{i} \mid X_{i}\right)} \geq \alpha\right] \leq C_{3} e^{-\alpha / C_{3}}
$$

for all $\alpha \geq C_{4} q^{2 r /(2 r+1)} n^{1 /(2 r+1)}$. 
(b).

$$
\varlimsup_{n \rightarrow \infty} \sup _{f \in \mathcal{M}_{q}} \frac{1}{n} \sum_{i=1}^{n} \log \frac{f\left(Y_{i} \mid X_{i}\right)}{f^{*}\left(Y_{i} \mid X_{i}\right)}<0, \text { a.s. }
$$

Proof. Let $h(y, x)=f(y \mid x) f_{X}(x)$ and $h^{*}(y, x)=f^{*}(y \mid x) f_{X}(x)$ for $f \in \mathcal{M}_{q}$ and $f^{*} \in \mathcal{M}_{q^{*}}$. Denote by $\bar{h}=\left(h+h^{*}\right) / 2$. We first have the inequalities $\mathcal{K}\left(h, h^{*}\right) \geq \mathcal{H}^{2}\left(h, h^{*}\right)$, and

$$
\sum_{i=1}^{n} \log \frac{h}{h^{*}} \leq 2 \sqrt{n} \nu_{n}\left(\log \frac{\bar{h}}{h^{*}}\right)-2 n \mathcal{K}\left(\bar{h}, h^{*}\right)
$$

Therefore,

$$
\begin{aligned}
\mathbb{P}\left[\sup _{h} \sum_{i=1}^{n} \log \frac{h}{h^{*}} \geq \alpha\right] & \leq \mathbb{P}\left[\sup _{h} \sqrt{n} \nu_{n}\left(\log \frac{\bar{h}}{h^{*}}\right)-n \mathcal{H}^{2}\left(\bar{h}, h^{*}\right) \geq \frac{\alpha}{2}\right] \\
& \leq \sum_{s=0}^{S} \mathbb{P}\left[\sup _{h \in \mathcal{G}_{s}} \nu_{n}\left(\log \frac{\bar{h}}{h^{*}}\right) \geq \frac{\alpha 2^{s-1}}{\sqrt{n}}\right] \\
& =\sum_{s=0}^{S} \mathbb{P}\left[\sup _{h \in \mathcal{G}_{s}} \nu_{n}\left(\log \sqrt{\frac{\bar{h}}{h^{*}}}\right) \geq \frac{\alpha 2^{s-2}}{\sqrt{n}}\right]
\end{aligned}
$$

where $\mathcal{G}_{0}=\left\{\bar{h}: n \mathcal{H}^{2}\left(\bar{h}, h^{*}\right) \leq \alpha\right\}, \mathcal{G}_{s}=\left\{\bar{h}: \alpha 2^{s-1}<n \mathcal{H}^{2}\left(\bar{h}, h^{*}\right) \leq \alpha 2^{s}\right\}, 1 \leq s \leq S, S=$ $\min \left\{s: \alpha 2^{s}>2 n\right\}$. We need to find the bracketing number for $\bar{H}_{q}(\epsilon)=\left\{\sqrt{\bar{h} / h^{*}}: \mathcal{H}\left(\bar{h}, h^{*}\right) \leq \epsilon\right\}$. This can be easily deduced from Lemma 1 such that

$$
N_{[]}\left(\bar{H}_{q}(\epsilon), \delta\right) \leq\left(\frac{2 \sqrt{2} C_{1} \epsilon}{\delta}\right)^{10\left(C_{2} \delta^{-\frac{1}{r}}+1\right) q+1}
$$

Note that $\int_{0}^{\epsilon} \sqrt{\log N_{[]}\left(\bar{H}_{q}(\epsilon), \delta\right)} d \delta \leq c \sqrt{q} \epsilon^{1-1 /(2 r)}$. It requires that $c \sqrt{q} \epsilon^{1-1 /(2 r)} \leq \sqrt{n} \epsilon^{2}$ such as $\epsilon \geq c_{1}(q / n)^{r /(2 r+1)}$. Next, apply Theorem 7.4 of van de Geer 2000$)$, together with $\sqrt{\alpha} 2^{s / 2+2} / \sqrt{n} \geq c_{1}(q / n)^{r /(2 r+1)}$, i.e., $\alpha \geq c_{2} q^{2 r /(2 r+1)} n^{1 /(2 r+1)}$. We obtain

$$
\sum_{s=0}^{S} \mathbb{P}\left[\sup _{h \in \mathcal{G}_{s}} \nu_{n}\left(\log \sqrt{\frac{\bar{h}}{h^{*}}}\right) \geq \frac{\alpha 2^{s-2}}{\sqrt{n}}\right] \leq \sum_{s=0}^{S} c_{3} e^{-\frac{\alpha 2^{s}}{c_{3} 2^{8}}} \leq C_{3} e^{-\alpha / C_{3}} .
$$


This finishes the proof of Part (a).

To prove Part (b), it follows from that it is enough to show that

$$
\lim _{n \rightarrow \infty} \sup _{f \in \mathcal{M}_{q}} n^{-1 / 2} \nu_{n}\left(\log \sqrt{\frac{\bar{h}}{h^{*}}}\right)=0, \text { a.s. }
$$

As in the proof of Part (a), we have

$$
\mathbb{P}\left[\sup _{f \in \mathcal{M}_{q}} n^{-1 / 2} \nu_{n}\left(\log \sqrt{\frac{\bar{h}}{h^{*}}}\right) \geq \alpha\right] \leq c_{4} e^{-n \alpha^{2} / c_{4}}
$$

for every $\alpha>0$ such that $c_{2} q^{2 r /(2 r+1)} n^{1 /(2 r+1)} \leq \alpha \sqrt{n} \leq 32 \sqrt{n}$. Hence,

$$
\sum_{n=1}^{\infty} \mathbb{P}\left[\sup _{f \in \mathcal{M}_{q}} n^{-1 / 2} \nu_{n}\left(\log \sqrt{\frac{\bar{h}}{h^{*}}}\right) \geq \alpha\right] \leq \infty
$$

for $0<\alpha \leq 32$. Part $\mathrm{b}$ follows from Borel-Cantelli.

Lemma 3. Assume B1 and B2 hold. Define

$$
\Delta_{n}\left(q, q^{*}\right)=\sup _{f \in \mathcal{M}_{q}}\left(\ell_{n}(f)-\lambda_{q, n}\|\beta\|_{K}^{2}\right)-\sup _{f \in \mathcal{M}_{q^{*}}}\left(\ell_{n}(f)-\lambda_{q^{*}, n}\|\beta\|_{K}^{2}\right)
$$

where $\lambda_{q, n}=C_{5} q^{2 r /(2 r+1)} n^{1 /(2 r+1)}$ and $\lambda_{q^{*}, n}=\tilde{C}_{5}\left(q^{*}\right)^{2 r /(2 r+1)} n^{1 /(2 r+1)}$. Then,

$$
\varlimsup_{n \rightarrow \infty} \sup _{q>q^{*}} \frac{\Delta_{n}\left(q, q^{*}\right)}{q^{\frac{2 r}{2 r+1}} n^{\frac{1}{2 r+1}}} \leq C_{6}, \quad \text { a.s. }
$$

Proof. Using the fact that

$$
\sup _{f \in \mathcal{M}_{q^{*}}}\left(\ell_{n}(f)-\lambda_{q^{*}, n}\|\beta\|_{K}^{2}\right) \geq \ell_{n}\left(f^{*}\right)-\lambda_{q^{*}, n}\left\|\beta^{*}\right\|_{K}^{2}
$$

yield

$$
\Delta_{n}\left(q, q^{*}\right) \leq \sup _{f \in \mathcal{M}_{q}} \sum_{i=1}^{n} \log \frac{f\left(Y_{i} \mid X_{i}\right)}{f^{*}\left(Y_{i} \mid X_{i}\right)}+\lambda_{q^{*}, n}\left\|\beta^{*}\right\|_{K}^{2}
$$


Furthermore, from Part (a) of Lemma 2 for $\alpha \geq C_{4}$,

$$
\mathbb{P}\left[\sup _{q \geq q^{*}} \frac{1}{q^{\frac{2 r}{2 r+1}} n^{\frac{1}{2 r+1}}} \sup _{f \in \mathcal{M}_{q}} \sum_{i=1}^{n} \log \frac{f\left(Y_{i} \mid X_{i}\right)}{f^{*}\left(Y_{i} \mid X_{i}\right)} \geq \alpha\right] \leq \sum_{q=q^{*}}^{\infty} C_{3} e^{-\alpha q^{\frac{2 r}{2 r+1}} n^{\frac{1}{2 r+1}} / C_{3}} \leq \frac{c_{1}}{n^{2}}
$$

The lemma follows easily using the Borel-Cantelli.

\section{A2: Proof of Theorem 1}

The constants $c_{i}$ used in the proof are all generic positive constants. First realize that any lower bound for a specific case yields immediately a lower bound for the general case. In the following, consider a special case where $q^{*}$ and $\left(\pi_{1}^{*}, \ldots, \pi_{q^{*}}^{*}\right)$ are known. $f_{0}$ is a fixed known density function.

Direct calculation of the Kullback-Leibler divergence between $h^{*}=f^{*}(y \mid x) f_{X}(x)$ and $h=f(y \mid x) f_{X}(x)$ where $f^{*}(y \mid x), f(y \mid x) \in \mathcal{M}_{q^{*}}$ yields

$$
\begin{aligned}
\mathcal{K}\left(h, h^{*}\right) & =\mathbb{E}_{h^{*}}\left\{\log \frac{h^{*}}{h}\right\}=\mathbb{E}_{h^{*}}\left\{\log \frac{\sum_{k=1}^{q^{*}} \pi_{k}^{*} h_{k}^{*}}{\sum_{k=1}^{q^{*}} \pi_{k}^{*} h_{k}}\right\}=\mathbb{E}_{h^{*}}\left\{\log \sum_{k=1}^{q^{*}} \tau_{k} \frac{h_{k}^{*}}{h_{k}}\right\} \\
& \geq \mathbb{E}_{h^{*}}\left\{\sum_{k=1}^{q^{*}} \tau_{k} \log \frac{h_{k}^{*}}{h_{k}}\right\}=\sum_{k=1}^{q^{*}} \pi_{k}^{*} \mathbb{E}_{h_{k}^{*}}\left\{\frac{h_{k} / h}{h_{k}^{*} / h^{*}} \log \frac{h_{k}^{*}}{h_{k}}\right\},
\end{aligned}
$$

where $\tau_{k}=\pi_{k}^{*} h_{k} / h$ for $k=1, \ldots, q^{*}$ and the inequality is due to the Jensen's Inequality. Note that $\pi_{k}^{*} h_{k} / h=\pi_{k}^{*} f_{0}\left(y-\eta_{k}\right) / \sum_{i=1}^{q^{*}} \pi_{i}^{*} f_{0}\left(y-\eta_{i}\right)$ is the probability of a data point coming from the $k$-th component. We assume this probability bound below away from zero. Therefore, $h_{k} / h$ 
is bounded below away from zero and above by a positive constant. Hence,

$$
\begin{aligned}
\mathcal{K}\left(h, h^{*}\right) & =\sum_{k=1}^{q^{*}} \pi_{k}^{*} \mathbb{E}_{h_{k}^{*}}\left\{\frac{h_{k} / h}{h_{k}^{*} / h^{*}} \log \frac{h_{k}^{*}}{h_{k}}\right\} \\
& =\sum_{k=1}^{q^{*}} \pi_{k}^{*} \int_{h_{k}^{*}>h_{k}} \frac{h_{k} / h}{h_{k}^{*} / h^{*}} h_{k}^{*} \log \frac{h_{k}^{*}}{h_{k}} d \mu+\sum_{k=1}^{q^{*}} \pi_{k}^{*} \int_{h_{k}^{*} \leq h_{k}} \frac{h_{k} / h}{h_{k}^{*} / h^{*}} h_{k}^{*} \log \frac{h_{k}^{*}}{h_{k}} d \mu, \\
& \geq c_{3} \sum_{k=1}^{q^{*}} \pi_{k}^{*} \int_{h_{k}^{*}>h_{k}} h_{k}^{*} \log \frac{h_{k}^{*}}{h_{k}} d \mu+c_{4} \sum_{k=1}^{q^{*}} \pi_{k}^{*} \int_{h_{k}^{*} \leq h_{k}} h_{k}^{*} \log \frac{h_{k}^{*}}{h_{k}} d \mu \\
& \geq c_{5} \sum_{k=1}^{q^{*}} \pi_{k}^{*} \mathcal{K}\left(h_{k}, h_{k}^{*}\right) .
\end{aligned}
$$

Using the fact $\log y-\log x-\frac{1}{x}(y-x)+\frac{1}{2 c_{6}}(y-x)^{2} \leq 0$ for $0 \leq y \leq \sqrt{c_{6}}$,

$$
\begin{aligned}
\mathcal{K}\left(h_{k}, h_{k}^{*}\right) & =\mathbb{E}_{h_{k}^{*}}\left\{\log \frac{h_{k}^{*}}{h_{k}}\right\} \\
& \geq \frac{1}{2 c_{6}} \mathbb{E}_{h_{k}^{*}}\left\{f_{0}\left(Y-\eta\left(X, \beta_{k}\right)\right)-f_{0}\left(Y-\eta\left(X, \beta_{k}^{*}\right)\right)\right\}^{2} \\
& =\frac{1}{2 c_{6}} \mathbb{E}_{h_{k}^{*}}\left\{\dot{f}_{0}{ }^{2}\left(Y-\tilde{\eta}_{k}\right)\left(\eta\left(X, \beta_{k}-\beta_{k}^{*}\right)\right)^{2}\right\} \\
& =\frac{1}{2 c_{6}} \mathbb{E}_{X}\left\{\mathbb{E}_{f_{0 k}(y \mid x)}\left(\dot{f}_{0}{ }^{2}\left(Y-\tilde{\eta}_{k}\right) \mid X\right)\left(\eta\left(X, \beta_{k}-\beta_{k}^{*}\right)\right)^{2}\right\} \\
& \geq c_{7} \mathbb{E}_{X}\left\{\left(\eta\left(X, \beta_{k}-\beta_{k}^{*}\right)\right)^{2}\right\} \\
& =c_{7}\left\|\beta_{k}-\beta_{k}^{*}\right\|_{C}^{2},
\end{aligned}
$$

where $\tilde{\eta}_{k}$ is a point between $\eta\left(X, \beta_{k}^{*}\right)$ and $\eta\left(X, \beta_{k}\right)$. Therefore, $\mathcal{K}\left(h, h^{*}\right)$ is bounded below by $\left\|\beta-\beta^{*}\right\|_{C}^{2}$ up to a constant, where $\left\|\beta-\beta^{*}\right\|_{C}^{2}=\sum_{k=1}^{q^{*}}\left\|\beta_{k}-\beta_{k}^{*}\right\|_{C}^{2}$. A similar calculation also yields that $\mathcal{K}\left(h, h^{*}\right)$ is bounded above by $\left\|\beta-\beta^{*}\right\|_{C}^{2}$ up to a constant.

In the following, we adopt the results from Tsybakov 2009$)$ to establish the lower bound, which is based upon testing multiple hypotheses. In particular, we can find a subset $\left\{\beta^{(0)}, \ldots, \beta^{(N)}\right\} \subset$ 


\section{REFERENCES}

$\mathcal{F}^{q^{*}}$ with $N$ increasing with $n$, such that for some positive constant $c$ and all $0 \leq i<j \leq N$,

$$
\left\|\beta^{(i)}-\beta^{(j)}\right\|_{C}^{2} \geq 2 c \gamma^{\frac{2 r}{2 r+1}}\left(n / q^{*}\right)^{-\frac{2 r}{2 r+1}}
$$

and

$$
\frac{1}{N} \sum_{j=1}^{N} \mathcal{K}\left(h^{(j)}, h^{(0)}\right) \leq \gamma \log N
$$

then we can conclude according to Theorem 2.5 of Tsybakov (2009) that,

$$
\inf _{\hat{\beta}} \sup _{\beta^{*} \in \mathcal{F}^{*}} \mathbb{P}\left(\left\|\beta^{(i)}-\beta^{(j)}\right\|_{C}^{2} \geq c \gamma^{\frac{2 r}{2 r+1}}\left(n / q^{*}\right)^{-\frac{2 r}{2 r+1}}\right) \geq \frac{\sqrt{N}}{1+\sqrt{N}}\left(1-2 \gamma-\sqrt{\frac{2 \gamma}{\log N}}\right)
$$

which yields

$$
\lim _{a \rightarrow 0} \lim _{n \rightarrow \infty} \inf _{\hat{\beta}} \sup _{\beta^{*} \in \mathcal{F}^{*}} \mathbb{P}\left(\left\|\hat{\beta}-\beta^{*}\right\|_{C} \geq a\left(n / q^{*}\right)^{-\frac{r}{2 r+1}}\right) \geq 1
$$

Hence Theorem 1 will be proved.

Next, we construct the subset $\left\{\beta^{(0)}, \ldots, \beta^{(N)}\right\} \subset \mathcal{F}^{q^{*}}, k=1, \ldots, q^{*}$. Let $\beta^{(j)}=\left(\beta_{1}^{(j)}, \beta_{2}^{(j)}, \ldots, \beta_{q^{*}}^{(j)}\right)$, $j=1, \ldots, N$. We show that both 4.26 and 4.27 are satisfied. Let $\widetilde{M}=\left\lfloor M / q^{*}\right\rfloor$ for some large number $M$ to be decided later. Consider the function space

$$
\mathcal{H}^{*}=\left\{\beta=\sum_{k=\widetilde{M}+1}^{2 \widetilde{M}} b_{k} M^{-1 / 2} L_{K^{1 / 2}} \varphi_{k}:\left(b_{M+1}, \ldots b_{2 M}\right) \in\{0,1\}^{M}\right\}
$$

where $\left\{\varphi_{k}: k \geq 1\right\}$ are the orthonomal eigenfunctions of $K^{1 / 2} C K^{1 / 2}$. For any $\beta \in \mathcal{H}^{*}$, observe 


\section{REFERENCES}

that

$$
\begin{aligned}
\|\beta\|_{K}^{2} & =\left\|\sum_{k=\widetilde{M}+1}^{2 \widetilde{M}} b_{k} M^{-1 / 2} L_{K^{1 / 2}} \varphi_{k}\right\|_{K}^{2} \\
& =\sum_{k=\widetilde{M}+1}^{2 \widetilde{M}} b_{k}^{2} M^{-1}\left\|L_{K^{1 / 2}} \varphi_{k}\right\|_{K}^{2} \\
& \leq \sum_{k=\widetilde{M}+1}^{2 \widetilde{M}} M^{-1}\left\|L_{K^{1 / 2}} \varphi_{k}\right\|_{K}^{2} \leq 1
\end{aligned}
$$

which shows that $\mathcal{H}^{*} \subset \mathcal{F}$. The Varshamov-Gilbert bound shows that for any $M \geq 8$, there exists a set $\mathcal{B}=\left\{b^{(0)}, b^{(1)}, \ldots, b^{(N)}\right\} \subset\{0,1\}^{M}$ such that

1. $b^{(0)}=(0, \ldots, 0)^{\prime}$

2. $H\left(b, b^{\prime}\right)>M / 8$ for any $b \neq b^{\prime} \in \mathcal{B}$, where $H(\cdot, \cdot)=\frac{1}{4} \sum_{i=1}^{M}\left(b_{i}-b_{i}^{\prime}\right)^{2}$ is the Hamming distance;

3. $N \geq 2^{M / 8}$.

The subset $\left\{\beta^{(0)}, \ldots, \beta^{(N)}\right\} \subset \mathcal{F}^{q^{*}}$ is chosen as $\beta_{j}^{(i)}=\sum_{k=\widetilde{M}+1}^{2 \widetilde{M}} b_{j, k-\widetilde{M}}^{(i)} M^{-1 / 2} L_{K^{1 / 2}} \varphi_{k}, i=$ $0, \ldots N, j=1, \ldots, q^{*}$. For any $0 \leq i<j \leq N$, observe that

$$
\left\|\beta^{(i)}-\beta^{(j)}\right\|_{C}^{2}=\sum_{l=1}^{q^{*}} \mathbb{E}_{X}\left(\left\langle\beta_{l}^{(i)}-\beta_{l}^{(i)}, X\right\rangle\right)^{2}=\sum_{l=1}^{q^{*}} \sum_{k=\widetilde{M}+1}^{2 \widetilde{M}}\left(b_{l, k-\widetilde{M}}^{(i)}-b_{l, k-\widetilde{M}}^{(j)}\right)^{2} M^{-1} \rho_{k} .
$$

Therefore,

$$
\left\|\beta^{(i)}-\beta^{(j)}\right\|_{C}^{2} \geq s_{2 \widetilde{M}} M^{-1} \sum_{l=1}^{q^{*}} \sum_{k=1}^{\widetilde{M}}\left(b_{l, k}^{(i)}-b_{l, k}^{(j)}\right)^{2} \geq \rho_{2 \widetilde{M}} / 2 \asymp\left(M / q^{*}\right)^{-2 r},
$$

and

$$
\left\|\beta^{(i)}-\beta^{(j)}\right\|_{C}^{2} \leq s_{\widetilde{M}} M^{-1} \sum_{l=1}^{q^{*}} \sum_{k=1}^{\widetilde{M}}\left(b_{l, k}^{(i)}-b_{l, k}^{(j)}\right)^{2} \leq \rho_{\widetilde{M}} \asymp\left(M / q^{*}\right)^{-2 r} .
$$




\section{REFERENCES}

By taking $M$ to be the smallest integer greater than $c_{2} \gamma^{-\frac{1}{2 r+1}}\left(q^{*}\right)^{2 r /(2 r+1)} n^{\frac{1}{2 r+1}}$ with $c_{2}=$ $\left(c_{1} \cdot 8 \log 2\right)^{1 /(1+2 r)}$, the theorem is proved.

\section{A3: Proof of Theorem 2}

For any $q \geq q^{*}$, since $\hat{f}$ is the maximum, we have

$$
-\ell(\hat{f})+\lambda\|\hat{\beta}\|_{K}^{2} \leq-\ell\left(f^{*}\right)+\lambda\left\|\beta^{*}\right\|_{K}^{2}
$$

which gives

$$
-\left(\ell(\hat{f})-\ell\left(f^{*}\right)\right)+\lambda\|\hat{\beta}\|_{K}^{2} \leq \lambda\left\|\beta^{*}\right\|_{K}^{2} .
$$

Define

$$
d_{h}=\frac{\sqrt{\frac{h}{h^{*}}}-1}{\mathcal{H}\left(h, h^{*}\right)}
$$

Using $\log (1+x) \leq x$,

$$
\begin{aligned}
\ell(f)-\ell\left(f^{*}\right) & =\sum_{i=1}^{n} 2 \log \left(1+\mathcal{H}\left(h, h^{*}\right) d_{h}\left(Y, X_{i}\right)\right) \\
& \leq \sum_{i=1}^{n} 2 \mathcal{H}\left(h, h^{*}\right) d_{h}\left(Y, X_{i}\right) \\
& =2 \sqrt{n} \nu_{n}\left(d_{h}\right) \mathcal{H}\left(h, h^{*}\right)-n \mathcal{H}^{2}\left(h, h^{*}\right),
\end{aligned}
$$

where $\nu_{n}(g)=n^{-1 / 2} \sum_{i=1}^{n}\left(g\left(Y_{i}, X_{i}\right)-\mathbb{E} g\left(Y_{i}, X_{i}\right)\right)$. So,

$$
-\left(\ell(f)-\ell\left(f^{*}\right)\right) \geq n \mathcal{H}^{2}\left(h, h^{*}\right)-2 \sqrt{n} \nu_{n}\left(d_{h}\right) \mathcal{H}\left(h, h^{*}\right) .
$$


Combining this with 4.29 ,

$$
\begin{aligned}
n \mathcal{H}^{2}\left(\hat{h}, h^{*}\right)+\lambda\|\hat{\beta}\|_{K}^{2} & \leq \lambda\left\|\beta^{*}\right\|_{K}^{2}+2 \sqrt{n} \nu_{n}\left(d_{\hat{h}}\right) \mathcal{H}\left(\hat{h}, h^{*}\right) \\
& =\lambda\left\|\beta^{*}\right\|_{K}^{2}+2 \sqrt{n}\left(\nu_{n}\left(g_{\hat{h}}\right)-\nu_{n}\left(g_{h^{*}}\right)\right),
\end{aligned}
$$

where $g_{h}=\sqrt{h / h^{*}}=\sqrt{f / f^{*}}$. It is critical to investigate the behavior of $\left|\nu_{n}\left(g_{h}\right)-\nu_{n}\left(g_{h^{*}}\right)\right|$ as a function of $\mathcal{H}\left(h, h^{*}\right)$.

It follows from Lemma 1 that the bracketing entropy of $H_{q}(\epsilon)$ is

$$
H_{[]}(\delta)=\log N_{[]}\left(H_{q}(\epsilon), \delta\right) \leq\left(10\left(C_{2} \delta^{-\frac{1}{r}}+1\right) q+1\right) \log \left(\frac{C_{1} \epsilon}{\delta}\right)
$$

Then,

$$
\int_{0}^{\epsilon} H_{[]}^{1 / 2}(\delta) d \delta \leq c \sqrt{q} \epsilon^{1-\frac{1}{2 r}}
$$

The reminder of proof is identical to that in Section 5.6 of van de Geer (2001). We obtain

$$
\sup _{f \in \mathcal{M}_{q}} \frac{\left|\nu_{n}\left(g_{h}\right)-\nu_{n}\left(g_{h^{*}}\right)\right|}{\sqrt{q} \mathcal{H}\left(h, h^{*}\right)^{1-\frac{1}{2 r}} \vee n^{-\frac{r-1 / 2}{2 r+1}}}=O_{p}(1)
$$

This allows us to conclude that

$$
\sqrt{n}\left|\nu_{n}\left(g_{\hat{h}}\right)-\nu_{n}\left(g_{h^{*}}\right)\right|=\sqrt{n} \frac{\left|\nu_{n}\left(g_{\hat{h}}\right)-\nu_{n}\left(g_{h^{*}}\right)\right|}{\sqrt{q} \mathcal{H}\left(\hat{h}, h^{*}\right)^{1-1 / 2 r}} \sqrt{q} \mathcal{H}\left(\hat{h}, h^{*}\right)^{1-1 / 2 r}=O_{p}(\sqrt{n}) \sqrt{q} \mathcal{H}\left(\hat{h}, h^{*}\right)^{1-1 / 2 r}
$$

Combining this with 4.30 yields that $\mathcal{H}\left(\hat{h}, h^{*}\right)=O_{p}\left(n^{-r /(2 r+1)}\right)$ provided that $\lambda$ is of order $n^{1 /(2 r+1)}$. This finishes the proof of Theorem. 


\subsection{Proof of Theorem 3}

First note that

$$
\varlimsup_{n \rightarrow \infty} \sup _{q>q^{*}} \frac{\Delta_{n}\left(q, q^{*}\right)}{\operatorname{pen}_{n}(q)-\operatorname{pen}_{n}\left(q^{*}\right)} \leq \lim _{n \rightarrow \infty} \sup _{q>q^{*}} \frac{q^{\frac{2 r}{2 r+1}} n^{\frac{1}{2 r+1}}}{\operatorname{pen}_{n}(q)-\operatorname{pen}_{n}\left(q^{*}\right)} \varlimsup_{n \rightarrow \infty} \sup _{q>q^{*}} \frac{\Delta_{n}\left(q, q^{*}\right)}{q^{\frac{2 r}{2 r+1}} n^{\frac{1}{2 r+1}}}=0 .
$$

Therefore, for all $q>q^{*}$.

$$
\sup _{f \in \mathcal{M}_{q}}\left(\ell_{n}(f)-\lambda_{q, n}\|\beta\|_{K}^{2}\right)-\operatorname{pen}_{n}(q)<\sup _{f \in \mathcal{M}_{q^{*}}}\left(\ell_{n}(f)-\lambda_{q^{*}, n}\|\beta\|_{K}^{2}\right)-\operatorname{pen}_{n}\left(q^{*}\right) .
$$

This shows that $\varlimsup_{n \rightarrow \infty} \hat{q}_{n} \leq q^{*}$ a.s., which means that we do not asymptotically overestimate the order.

On the other hand, for any $q<q^{*}$,

$$
\varlimsup_{n \rightarrow \infty} \frac{1}{n} \Delta_{n}\left(q, q^{*}\right) \leq \varlimsup_{n \rightarrow \infty} \sup _{f \in \mathcal{M}_{q}} \frac{1}{n} \sum_{j=1}^{n} \log \frac{f\left(Y_{i} \mid X_{i}\right)}{f^{*}\left(Y_{i} \mid X_{i}\right)}+\lim _{n \rightarrow \infty} \frac{\lambda_{q^{*}, n}}{n}\left\|\beta^{*}\right\|_{K}^{2}
$$

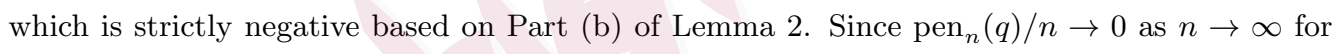
$q<q^{*}$, we have

$$
\varlimsup_{n \rightarrow \infty} \frac{1}{n}\left\{\Delta_{n}\left(q, q^{*}\right)-\operatorname{pen}_{n}(q)+\operatorname{pen}_{n}\left(q^{*}\right)\right\}<0, \text { a.s. }
$$

We obtain, for all $q<q^{*}$,

$$
\sup _{f \in \mathcal{M}_{q}}\left(\ell_{n}(f)-\lambda_{q, n}\|\beta\|_{K}^{2}\right)-\operatorname{pen}_{n}(q)<\sup _{f \in \mathcal{M}_{q^{*}}}\left(\ell_{n}(f)-\lambda_{q^{*}, n}\|\beta\|_{K}^{2}\right)-\operatorname{pen}_{n}\left(q^{*}\right) .
$$

This shows that $\varlimsup_{n \rightarrow \infty} \hat{q}_{n} \geq q^{*}$ a.s., which means that we do not asymptotically underestimate the order. 